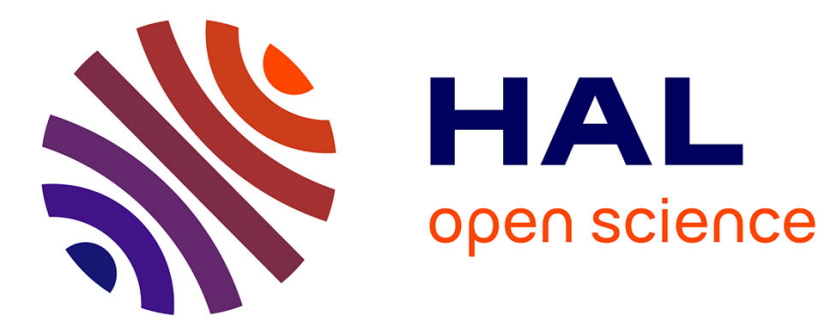

\title{
Maximal smoothings of real plane curve singular points.
}

Viatcheslav Kharlamov, Jean-Jacques Risler, Eugenii Shustin

\section{To cite this version:}

Viatcheslav Kharlamov, Jean-Jacques Risler, Eugenii Shustin. Maximal smoothings of real plane curve singular points.. 1998. hal-00129577

\section{HAL Id: hal-00129577 \\ https://hal.science/hal-00129577}

Preprint submitted on 8 Feb 2007

HAL is a multi-disciplinary open access archive for the deposit and dissemination of scientific research documents, whether they are published or not. The documents may come from teaching and research institutions in France or abroad, or from public or private research centers.
L'archive ouverte pluridisciplinaire HAL, est destinée au dépôt et à la diffusion de documents scientifiques de niveau recherche, publiés ou non, émanant des établissements d'enseignement et de recherche français ou étrangers, des laboratoires publics ou privés. 


\title{
Maximal smoothings of real plane curve singular points
}

\author{
V. Kharlamov * \\ IRMA et Université Louis Pasteur \\ 7, rue René-Descartes \\ 67084 Strasbourg Cedex \\ France \\ J.-J. Risler \\ Université de Paris 6 \\ Département de Mathématiques \\ 4 place Jussieu \\ 75252 Paris Cedex 05, France \\ E. Shustin * \\ Tel Aviv University \\ School of Mathematical Sciences \\ Ramat Aviv, 69978 Tel Aviv \\ Israel
}

To V.A.Rokhlin who guided certain of us in the marvelous world behind reals.

\begin{abstract}
The local Harnack inequality bounds from above the number of ovals which can appear in a small perturbation of a singular point. As is known, there are real singular points for which this bound is not sharp. We show that Harnack inequality is sharp in any complex topologically equisingular class: every real singular point is complex deformation equivalent to a real singularity for which Harnack inequality is sharp. For semi-quasi-homogeneous and some other singularities we exhibit a real deformation with the same property. A refined Harnack inequality and its sharpness are discussed as well.
\end{abstract}

*This work was started during the stay of the third author at Ecole Normale Supérieure and Univeristé de Strasbourg, continued during the stay of the first and third author at Universität Kaiserslautern and finished during the stay of the third author at the University of Toronto. We thank all these institutions for hospitality and financial support. 
The Harnack theorem [6] states that a real plane projective curve of degree $d$ has at most $\frac{1}{2}(d-1)(d-2)+1$ connected components, and for any degree $d$ curves with this number of components ( $M$-curves) do exist. Every $M$-curve of degree $d$ is nonsingular and can be considered as a nonsingular perturbation of an ordinary homogeneous singularity of multiplicity $d$ : pick a generic system of affine coordinates and consider the family $f_{\varepsilon}(x, y)=\varepsilon^{d} f\left(\varepsilon^{-1} x, \varepsilon^{-1} y\right)$, where $f=0$ is an equation of the curve.

Small nonsingular real perturbations (further on referred to as smoothings) of an isolated real plane curve singular point is subject to the local analogue of the Harnack bound: the number $v$ of closed components (further called ovals) of a smoothing satisfies the inequality

$$
2 v \leq\left\{\begin{array}{cc}
\mu-r+1, & \text { if the singularity has a real branch } \\
\mu-r+3, & \text { otherwise }
\end{array}\right.
$$

where $\mu$ is the Milnor number and $r$ the number of complex branches of the singular point (see $[1,16])$. The question on the sharpness of this estimate (i.e., on the existence of M-smoothings) turned to be more subtle than for projective curves: as it is proven in [16], (1) is sharp for unibranch singular points (see [15] for a different proof); on the other hand, there are singular points which have no M-smoothing (see $[9])$. It brings us before the task to describe the class of singular points for which (1) is sharp. The present paper is devoted to this problem (additional information is found in [10] and [4]; [4] contains some mistaken statements, easily recognizable, cf., remarks below).

We prove that for any singular point there exists a singular point which is topologically equivalent to it over $\mathbb{C}$ and has $M$-smoothing (more detailed statement is given in Theorem 1, section 3.2). Another result of the present paper (Theorem $2(2)$ in section 4) states that varying a real nondegenerate semi-quasi-homogeneous point through the real nondegenerate semi-quasi-homogeneous points one can obtain a point which has $M$-smoothing. The proofs are constructive. The proof of the first statement is based on a local version of the original Harnack construction for curves (cf., $[16,10])$. The proof of the second one is based on the Viro patchworking method, which is a far-reaching generalization of the Harnack one (see [22]). Under some additional hypothesis on the singular point the above methods provide M-smoothings without any preliminary equisingular deformation of the singularity (Proposition 1 in section 3.1 and Theorem 2(1,3) in section 4).

In addition, in section 5 we consider an improved local Harnack bound, which takes into account the arrangement of nonclosed components of smoothings, and we show that this improved bound is sharp in the real equisingular class of any Newton-nondegenerate singular point (Theorem 5 in section 5 ).

In the last section we discuss some open problems.

Acknowledgment. We are grateful to S. Orevkov for stimulating discussions: Theorem 1 answers one of his questions. 


\subsection{Smoothings of singular points}

A holomorphic curve $C$ in $\mathbb{C}^{2}$ or in another complex surface with complex conjugation is called real, if $C$ is invariant under complex conjugation. A real isolated singular point is a germ $(C, z) \subset\left(\mathbb{C}^{2}, z\right), z \in \mathbb{R}^{2}$, of a real plane reduced holomorphic curve $C$. With usual for isolated singularities ambiguity, we denote by $B(C, z)$ a Milnor ball (see [14] or [2]; recall that each branch of $C$ meets $\partial B(C, z)$ transversally and along a smooth circle) whose radius we diminish, if necessary, when making perturbations.

A real holomorphic curve $C^{\prime}$ in the Milnor ball $B(C, z)$ is called a real smoothing of $C$ if there exists a real-analytic 1-parameter family $C_{t}, t \in[0,1]$, of real holomorphic curves in $B(C, z)$ such that $C_{0}=C, C^{\prime}=C_{1}$ and each $C_{t}$ with $t>0$ is nonsingular and transversal to the boundary of $B(C, z)$. We call such a family a smoothing out deformation of $(C, z)$.

The real part $C_{\mathbb{R}}^{\prime}$ of $C^{\prime}$ consists of finitely many ovals and nonclosed components. The number of nonclosed components is equal to the number $r_{\mathbb{R}}$ of real branches of $(C, z)$. The number $v$ of the ovals satisfies (1), and $C^{\prime}$ is called an $M$-smoothing if (1) turns into equality, i.e., $v=\frac{1}{2}(\mu-r+1)$ if $r_{\mathbb{R}}>0$, and $v=\frac{1}{2}(\mu-r+3)$ if $r_{\mathbb{R}}=0$.

Recall that $\frac{1}{2}(\mu-r+1)$ is equal to the genus of smoothings (as well as to the number $\delta$ of virtual double points, which is the maximal number of double points appearing in small perturbations of the singularity, diminished by $r-1$ ). If an isolated plane curve singular point is given by a polynomial or power series equation $f=0$ and the truncations of $f$ to the edges of the Newton diagram $\Gamma(f)$ of $f$ have no critical points in $\left(\mathbb{C}^{*}\right)^{2}$, then

$$
\frac{1}{2}(\mu-r+1)=\#\left(\operatorname{Int}(\mathcal{D}) \cap \mathbb{Z}^{2}\right)
$$

where $\mathcal{D}$ is the domain bounded in $\mathbb{R}^{2}$ by $\Gamma(f)$ and the coordinate axes and Int states for the interior (this formula can be found already in [3], for far-reaching generalizations and a modern exposition see $[11,12])$. Singular points satisfying the hypothesis of the above statement are called Newton nondegenerate (shortly ND).

An isolated singular point $(C, 0)$ is called semi-quasi-homogeneous if in some local coordinates it is given by an equation $f=0$ such that the truncation $f^{\gamma}$ of $f$ to an edge $\gamma$ of $\Gamma(f)$ has no critical points in $\mathbb{C}^{2} \backslash\{(0,0)\}$. It is equivalent to

$$
f^{\gamma}(x, y)=\alpha x^{a} y^{b} \prod_{i=1}^{s}\left(y^{c}-\alpha_{i} x^{d}\right)
$$

where: $c$ and $d$ are coprime; $\alpha_{1}, \ldots, \alpha_{s}$ are distinct and nonzero; $\alpha \neq 0$ and $a, b \in$ $\{0,1\}$. The numbers $\alpha_{1}, \ldots, \alpha_{s}$ are called peripheral roots of $f$. Note, that adding $x^{i}$ or $y^{j}$ with big $i, j$ we don't change the singularity up to isomorphism (see [21]) and, in particular, can make $\Gamma(f)$ compact, i.e., containing vertices on the both axes. 


\subsection{Topological equivalence of singular points}

We distinguish equivalences over $\mathbb{C}$ and over $\mathbb{R}$. A topological equivalence over $\mathbb{C}$ (resp., over $\mathbb{R}$ ) of two (real, in the case of the real equivalence) singular points $(C, z)$ and $(D, w)$ is a homeomorphism $\varphi: B(C, z) \rightarrow B(D, w)$ which takes $C \cap B(C, z)$ to $D \cap B(D, w)$ (and commutes with the complex conjugation in the real case). Singularities $(C, z)$ and $(D, z)$ are called topologically equisingular (or deformation equivalent) over $\mathbb{C}$ (over $\mathbb{R}$ ) if there exists a real-analytic family of topologically equivalent over $\mathbb{C}$ (resp., over $\mathbb{R}$ ) complex (resp., real) singularities $\left(C_{t}, z\right) \subset B(C, z)$, $t \in[0,1]$, connecting $(C, z)$ and $(D, z)$. As is known, a real-analytic family satisfies this property (both in the complex and real cases) if, and only if, the Milnor number is constant (see, for example, [13]).

Recall also the following known facts: (1) topologically equivalent isolated plane curve singularities are topologically equisingular over $\mathbb{C}$ (see [19]); (2) if two real unibranch singular points are topologically equivalent over $\mathbb{C}$, they are topologically equisingular over $\mathbb{R} ;(3)$ any real plane curve singular point is topologically equivalent over $\mathbb{C}$ to a singular point with all local branches real; (4) two real ND singular points are topologically equivalent over $\mathbb{R}$ if their equations have the same Newton diagram and for any edge of the diagram the truncations of the both have the same numbers of positive and negative peripheral roots; (5) replacing a real isolated singular point $f=0$ by $T f=0$ where $T f$ is the Taylor polynomial of $f$ of degree $\geq \mu+1$ we do not change the singularity up to real analytic coordinate transformation (see [21]).

\section{M-smoothings}

\subsection{Prélude: blowing-up construction}

The blowing-up method for construction of smoothings of an isolated real plane curve singularity is described in details in [16]. Here, we give an application of this method, which allow us to deliver some information on the class of singularities which have M-smoothing and to recall the principal ingredients of the method.

Proposition 1 Any singular point whose branches all are real and nonsingular, has M-smoothing.

Proof. We make induction by blow-ups introducing a stronger statement which takes into account the position of the smoothing with respect to a straight line.

Let $(C, z)$ be a singular point with $r$ branches which all are real and nonsingular, and $L$ be a real straight line through $z$ transversal to the branches of $C$. Let us fix an orientation of $L_{\mathbb{R}}$ and denote the components of $B(C, z)_{\mathbb{R}} \backslash L$ by $B_{+}$and $B_{-}$. We say that a real smoothing $C^{\prime}$ of $(C, z)$ is of type $(\varepsilon, \delta)$ with respect to $L$, where $\varepsilon, \delta= \pm 1$, if $C^{\prime}$ has a nonclosed real component which starts in $B_{\varepsilon}$, then successively intersects $L$ at $r$ points ordered in accordance with the fixed orientation of $L_{\mathbb{R}}$, and ends in $B_{\delta}$ (see Figure 1 ; it specifies also the convention distinguishing $B_{+}$and $B_{-}$). 
$\mathrm{B}_{+}$

$\mathrm{B}_{+}$

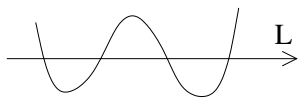

B.

type $(+1,+1)$

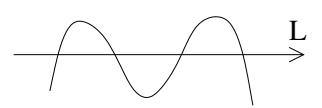

B

type $(-1,-1)$

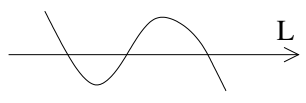

B.

type $(+1,-1)$

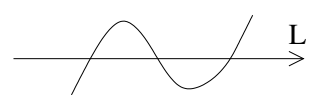

B.

type $(-1,+1)$

Figure 1: M-smoothings of a singular point with nonsingular branches

Prove that under the above hypothesis on $(C, z)$ and $L$, given $\varepsilon, \delta= \pm 1$ such that $\varepsilon \delta=(-1)^{r}$, there exists an M-smoothing of $(C, z)$ of type $(\varepsilon, \delta)$ with respect to $L$.

Proceed by induction on $\mu(C, z)$. If $\mu(C, z) \leq 1$ the statement is trivial, so assume that $\mu(C, z)>1$. Blow up the point $z$. The strict transform $C^{*}$ of $C$ intersects the exceptional divisor $E$ at real points $z_{1}, \ldots, z_{m}$, and all local branches of $C^{*}$ are real, nonsingular and transversal to $E$. Without loss of generality suppose that $\delta=+1$, and fix an orientation of $E_{\mathbb{R}}$ as in Figure 2a. By the induction assumption the singular points $\left(C^{*}, z_{1}\right),\left(C^{*}, z_{2}\right), \ldots,\left(C^{*}, z_{m}\right)$ have M-smoothings $C_{1}^{\prime}, \ldots, C_{m}^{\prime}$ of types $\left(+1, \varepsilon_{1}\right),\left(\varepsilon_{1}, \varepsilon_{2}\right), \ldots,\left(\varepsilon_{m-1}, \varepsilon_{m}\right)$, respectively, with respect to $E$ (see Figure $2 \mathrm{~b}$ ).

Now, to finish the proof use the recursive Harnack procedure. Pick up $r-1$ real holomorphic nonsingular curves $L_{1}^{(0)}, \ldots, L_{r-1}^{(0)}$ crossing $E$ transversally at $r-1$ distinct points positioned as in Figure $2 \mathrm{~b}$ (these curves are shown dashed), and deform the union of the smoothings $C_{1}^{\prime}, \ldots, C_{m}^{\prime}$ in the family $\widetilde{C}_{1}=C_{1}^{\prime} \ldots C_{m}^{\prime}+t L_{1}^{(0)} \ldots L_{r-1}^{(0)}$. If $t$ is small and of proper sign, the curve $\widetilde{C}_{1}$ has only one real component intersecting $E \cup L^{*}$; this component is nonclosed and crosses $E_{\mathbb{R}}$ at $r-1$ points and $L_{\mathbb{R}}^{*}$ at one point as shown in Figure 2c. In the next step pick up $r-2$ real holomorphic nonsingular curves $L_{1}^{(1)}, \ldots, L_{r-2}^{(1)}$ crossing $E$ transversally at $r-2$ distinct points positioned as in Figure 2c (these curves are shown dashed), and deform $\widetilde{C}_{1}$ in the family $\widetilde{C}_{1}+t L_{1}^{(1)} \ldots L_{r-2}^{(1)}$. Thus, one obtains a curve $\widetilde{C}_{2}$, whose only real component intersecting $E \cup L^{*}$ is non-closed, meets $E_{\mathbb{R}}$ at $r-2$ points and $L_{\mathbb{R}}^{*}$ at two points and is located as shown in Figure 2d. Repeat this procedure alternating the position of auxiliary curves with respect to $L^{*}$ until obtaining a curve $\widetilde{C}_{m}$ whose only real component intersecting $E \cup L^{*}$ does not meet $E_{\mathbb{R}}$. Then, this real component crosses $L_{\mathbb{R}}^{*}$ at $r$ points as shown in Figure 2 e. Blowing $E$ down transforms $\widetilde{C}_{m}$ into an $M$ smoothing of $(C, z)$ of type $(\varepsilon,+1)$ (computations similar to those done in the proof of Theorem 1 show that the total number of ovals obtained is $\left.\frac{1}{2}(\mu-r+1)\right)$.

Remark 1 A similar result, but for a more restricted class of singularities is contained in [4].

It may be interesting to study the class of M-smoothings obtained by the blowingup construction. More precisely, let us call a BM-smoothing an M-smoothing obtained by the algorithm proposed in [16] or by another inductive algorithm which depends only on the topology of the intermediate germs of curves with respect to the resolution trees. Then, as it follows from the simultaneous resolution theorem 


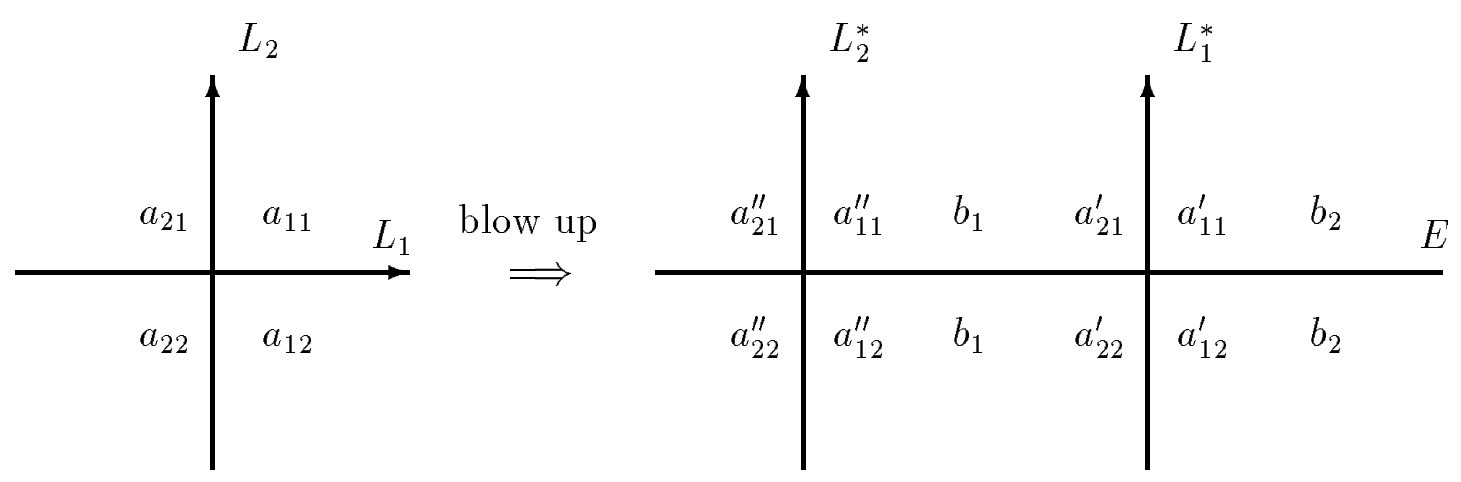

Figure 3: Blowing up a singular point

In the above notation, assume that $L_{1}, L_{2}$ are the coordinate axes in some local coordinate system $(x, y)$. For the singular point $(C, z)$ we define the $2 \times 2$ matrix $A(C, z)=\left(a_{i j}\right)_{i, j=1,2}$ over $\mathbb{Z} / 2 \mathbb{Z}$ so that $a_{i j}$ is the $\bmod 2$ residue of the number of the real demi-branches (components of $\left.C \cap\left(B(C, z) \mathbb{R}_{\mathbb{R}} \backslash\{z\}\right)\right)$ of $C$ in $\mathbb{R}_{i, j}^{2}=\left\{x(-1)^{i}<\right.$ $\left.0, y(-1)^{j}<0\right\}$.

Proposition 2 If $A(C, z) \neq 0$ then there is a germ $(D, z)$ such that $(C, z)$ and $(D, z)$ are $\left(L_{1} L_{2}\right)$-equivalent over $\mathbb{C}$ and

$$
A(D, z)=A(C, z)+J, \quad J=\left(\begin{array}{ll}
1 & 1 \\
1 & 1
\end{array}\right)
$$

Proof. A nonzero matrix $A(C, z)$ contains two or four units. If $A(C, z)$ has two units, one obtains the required germ $(D, z)$ by means of transformations $(x, y) \mapsto$ $(-x, y)$ and $(x, y) \mapsto(x,-y)$.

If all the entries of $A(C, z)$ are units we apply induction on the Milnor number $\mu(C, z)$. In the case $\mu(C, z)=1$, which corresponds to an ordinary node, it is sufficient to rotate one line (or to make a linear transformation).

Assume that $\mu(C, z)>1$ and blow up the point $z$. Denote by $E$ the exceptional divisor, by $U$ a neighborhood of $E_{\mathbb{R}}$ in the blown up $\mathbb{R}^{2}$, by $C^{*}, L_{1}^{*}, L_{2}^{*}$ the strict transforms of $C, L_{1}, L_{2}$, respectively, and by $z_{1}^{*}=E \cap L_{1}^{*}, z_{2}^{*}=E \cap L_{2}^{*}$ the intersection points. Denote by $a_{i j}^{\prime} \in \mathbb{Z} / 2 \mathbb{Z}$ (resp. by $\left.a_{i j}^{\prime \prime} \in \mathbb{Z} / 2 \mathbb{Z}\right), i, j=1,2$, the $\bmod 2$ residue of the number of real demi-branches of $\left(C^{*}, z_{1}^{*}\right)$ (resp. $\left.\left(C^{*}, z_{2}^{*}\right)\right)$ in the corresponding component of $U \backslash E_{\mathbb{R}}$ (see Figure 3 ). Finally, denote by $b_{i} \in \mathbb{Z} / 2 \mathbb{Z}, i=1,2$, the $\bmod 2$ residue of the number of real demi-branches of $C^{*}$ which are centered on $E_{\mathbb{R}} \backslash\left\{z_{1}^{*}, z_{2}^{*}\right\}$ and contained in the corresponding component of $U \backslash E_{\mathbb{R}}$ (see Figure 3 ). In the above notation

$$
A(C, z)=A^{\prime}+A^{\prime \prime}+\left(\begin{array}{ll}
b_{1} & b_{2} \\
b_{2} & b_{1}
\end{array}\right), \quad A^{\prime}=\left(\begin{array}{ll}
a_{21}^{\prime} & a_{11}^{\prime} \\
a_{12}^{\prime} & a_{22}^{\prime}
\end{array}\right), A^{\prime \prime}=\left(\begin{array}{ll}
a_{11}^{\prime \prime} & a_{22}^{\prime \prime} \\
a_{21}^{\prime \prime} & a_{12}^{\prime \prime}
\end{array}\right) .
$$

Since the Milnor numbers of the points of $C^{*}$ are strictly less than $\mu(C, z)$, the induction assumption applies to them. If $A^{\prime} \neq 0$ (or $A^{\prime \prime} \neq 0$ ), by induction one 

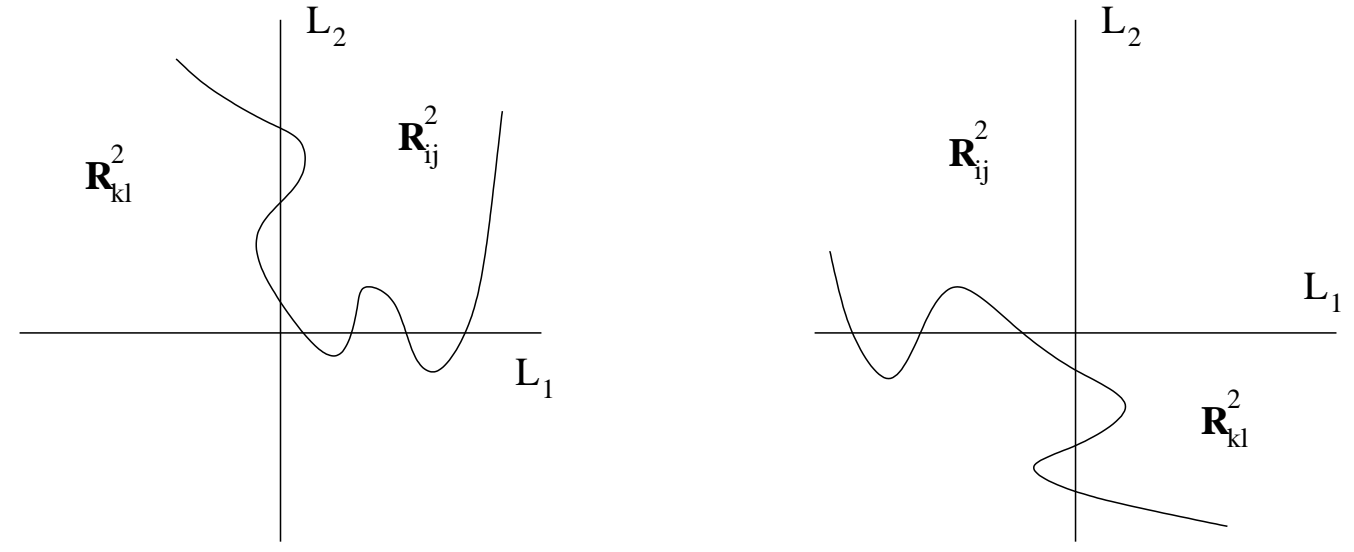

Figure 4: $(i j, k l)$-regular M-smoothing

can convert $A^{\prime}$ into $A^{\prime}+J$ (resp. $A^{\prime \prime}$ into $A^{\prime \prime}+J$ ), then blow down and obtain the required germ with $A(D, z)=0$. If $A^{\prime}=A^{\prime \prime}=0$ then at least one of $b_{1}$ and $b_{2}$ is equal to 1 , and in this case we move all the points $C^{*} \cap\left(E_{\mathbb{R}} \backslash\left\{z_{1}^{*}, z_{2}^{*}\right\}\right)$ along $E_{\mathbb{C}}$ into one interval of $E_{\mathbb{R}} \backslash\left\{z_{1}^{*}, z_{2}^{*}\right\}$ ), obtaining after blowing down the required germ.

Definition 2. Let $(C, z)$ be a singular point, $L_{1}, L_{2}$ be two distinct real straight lines through $z$ which are not components of $C$, and $n_{i}=\left(C \circ L_{i}\right)_{z}, i=1,2$, be the intersection numbers. Choose real local coordinates $x, y$ so that $L_{1}=\{y=0\}$, $L_{2}=\{x=0\}$ and assume that $A(C, z) \neq J$. An M-smoothing $C^{\prime}$ of $(C, z)$ is called $(i j, k l)$-regular (with respect to $L_{1}, L_{2}$ ), where $i, j, k, l, \in\{1,2\}$, if $C^{\prime}$ contains a nonclosed branch $\lambda$ which goes from $\mathbb{R}_{i j}^{2}$ to $\mathbb{R}_{k l}^{2}$ and, first, successively crosses $L_{1}$ at $n_{1}$ points $x_{s}, s=1, \ldots, n_{1}$, such that

$$
(-1)^{i} x_{1}>\ldots>(-1)^{i} x_{n_{1}}>0
$$

and then successively crosses $L_{2}$ at $n_{2}$ points $y_{s}, s=1, \ldots, n_{2}$, such that

$$
0<(-1)^{l} y_{1}<\ldots<(-1)^{l} y_{n_{2}}
$$

(see Figure 4). Clearly, $k-i \equiv n_{2} \bmod 2$ and $l-j \equiv n_{1} \bmod 2$.

Note that if there exists an M-smoothing $(i j, k l)$-regular with respect to two lines, then $A(C, z) \neq J$.

Proposition 2 and Proposition 3 below complete the proof of Theorem 1.

Proposition 3 Let $(C, z), L_{1}, L_{2}$ be as in Definition 2.

- If $A(C, z)=0$, there exists a singular point $(D, z)$ such that: $(C, z)$ and $(D, z)$ are $\left(L_{1} L_{2}\right)$-equivalent, $A(D, z)=0$ and $(D, z)$ has a $(11,11)$-regular M-smoothing.

- If $A(C, z) \neq J$ and $a_{i j}=a_{k l}=1$ (in particular, $(A(C, z) \neq 0$ ), there exists a singular point $(D, z)$ such that: $(C, z)$ and $(D, z)$ are $\left(L_{1} L_{2}\right)$-equivalent, $A(D, z)=A(C, z)$ and $(D, z)$ has an $(i j, k l)$-regular $M$-smoothing. 
at points on $E \backslash\left\{z_{1}^{*}, z_{2}^{*}\right\}$ (some of them may be empty).

In what follows we replace the germs of $C^{*}$ by $\left(E, L_{1}^{*}\right)^{-}$, or $\left(E, L_{2}^{*}\right)^{-}$, or $E$ equivalent germs, and move the germs $\left(C^{*}, w\right), w \in E \backslash\left\{z_{1}^{*}, z_{2}^{*}\right\}$, along $E \backslash\left\{z_{1}^{*}, z_{2}^{*}\right\}$. These operations give after blowing down a singular point $(D, z)$ which is $\left(L_{1}, L_{2}\right)$ equivalent to $(C, z)$ and has $A(D, z)=A(C, z)$ or $A(C, z)+J$. Then, according to the second observation above, it remains to construct a regular M-smoothing of $(D, z)$. (the case $(A(C, z)=0, A(D, z)=J)$ is excluded, since, as it is shown below, $(D, z)$ has a regular $\mathrm{M}$-smoothing).

Denote by $\left[z_{1}^{*}, z_{2}^{*}\right]$ the segment in $E_{\mathbb{R}}$ which is the common boundary of the images of $\mathbb{R}_{11}^{2}$ and $\mathbb{R}_{22}^{2}$ in the blown up plane (see Figure 3 ), and place all the germs of $C^{*}$ centered on $E_{\mathbb{R}} \backslash\left\{z_{1}^{*}, z_{2}^{*}\right\}$ to the interval $E_{\mathbb{R}} \backslash\left[z_{1}^{*}, z_{2}^{*}\right]$ so that the germs $\left(C^{*}, w\right)$ with odd intersection numbers $\left(C^{*} \circ E\right)_{w}$ are in a neighborhood of $z_{1}^{*}$ and the germs with even $\left(C^{*} \circ E\right)_{w}$ are in a neighborhood of $z_{2}^{*}$. For the sake of simplicity we use the same symbol $C^{*}$ in the notation of new germs.

Construction of a regular M-smoothing depends on the matrices $A\left(C^{*}, z_{1}^{*}\right)$, $A\left(C^{*}, z_{2}^{*}\right)$ with entries $a_{i j}^{\prime}, a_{i j}^{\prime \prime}$ distributed as shown in Figure 3 and the multiplicity $m$ of $(C, z)$, which is equal to the total intersection number of $C^{*}$ with $E$. By the induction assumption and according to Proposition 2 and the remarks made in the beginning of the proof, for any possible combination of $m, A\left(C^{*}, z_{1}^{*}\right), A\left(C^{*}, z_{2}^{*}\right)$, we can replace each germ $\left(C^{*}, w\right), w \in E$, with a suitable regular M-smoothing as shown in Figures 7,8 (changing $\left(C^{*}, w\right)$ if necessary in its topological $\left(E, L_{1}^{*}\right)^{-},\left(E, L_{2}^{*}\right)^{-}$, or $E$-equivalence class), where the triples $m, A\left(C^{*}, z_{1}^{*}\right), A\left(C^{*}, z_{2}^{*}\right)$ are encoded by symbols (odd, $A_{i}, A_{j}$ ) or (even, $A_{i}, A_{j}$ ) with

$$
\begin{gathered}
A_{0}=0, \quad A_{1}=\left(\begin{array}{ll}
1 & 1 \\
0 & 0
\end{array}\right) \text { or }\left(\begin{array}{ll}
0 & 0 \\
1 & 1
\end{array}\right), \\
A_{2}=\left(\begin{array}{ll}
0 & 1 \\
1 & 0
\end{array}\right) \text { or }\left(\begin{array}{ll}
1 & 0 \\
0 & 1
\end{array}\right), \quad A_{3}=\left(\begin{array}{ll}
1 & 0 \\
1 & 0
\end{array}\right) \text { or }\left(\begin{array}{ll}
0 & 1 \\
0 & 1
\end{array}\right) .
\end{gathered}
$$

Denote the union of the smoothed germs by $\widetilde{C}_{0}$.

Since $\delta=\frac{1}{2}(\mu+r-1)$ drops by $\frac{1}{2} m(m-1)$ when blowing up (see [7]), the total number of ovals in $\widetilde{C}_{0}$ is

$$
\begin{gathered}
v_{0}=\sum_{w \in E} \frac{\mu\left(C^{*}, w\right)-r\left(C^{*}, w\right)+1}{2} \\
=\frac{\mu(C, z)-r(C, z)+1}{2}+\#\left(C^{*} \cap E\right)-1-\frac{m(m-1)}{2} .
\end{gathered}
$$

Next we deform $\widetilde{C}_{0}$ in the following $m$-step recursive procedure. At the first step, we vary $\widetilde{C}_{0} \cup E$ in a linear pencil generated by $\widetilde{C}_{0} \cup E$ and a real divisor intersecting $E$ at $m-1$ points. Namely, in terms of the equations in an affine chart of $U$, we define $\widetilde{C}_{1}=E \widetilde{C}_{0}+t L_{1}^{(0)} \ldots L_{m-1}^{(0)}$, where $t$ is a small real parameter and $L_{1}^{(0)}, \ldots, L_{m-1}^{(0)}$ are real holomorphic curves crossing $E$ transversally at $m-1$ distinct points in the interior of $\left[z_{1}^{*}, z_{2}^{*}\right]$ (one can take the strict transforms of appropriate straight lines 


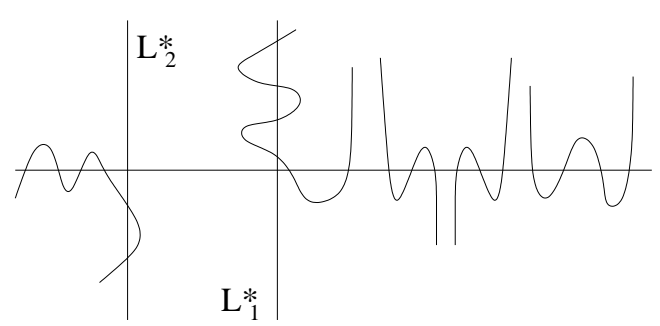

(even, $A_{0}, A_{0}$ )

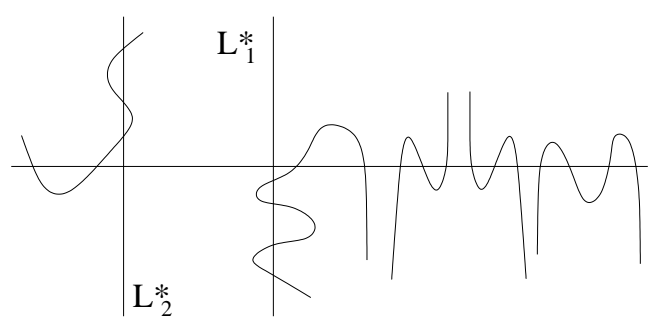

(even, $\left.A_{0}, A_{1}\right)$

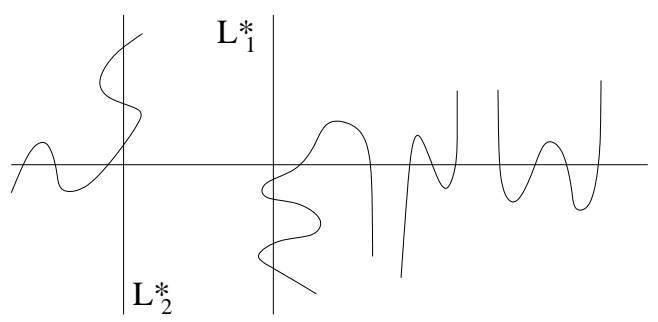

(even, $\left.A_{0}, A_{2}\right)$

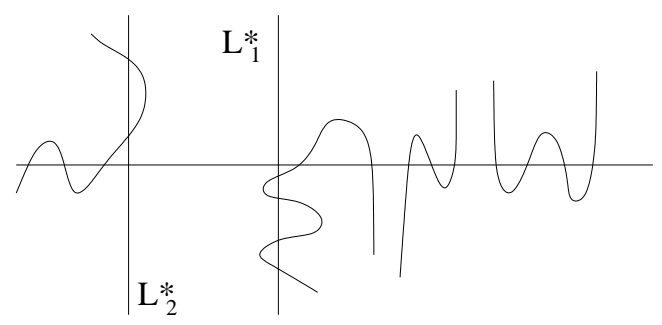

(even, $\left.A_{0}, A_{3}\right)$

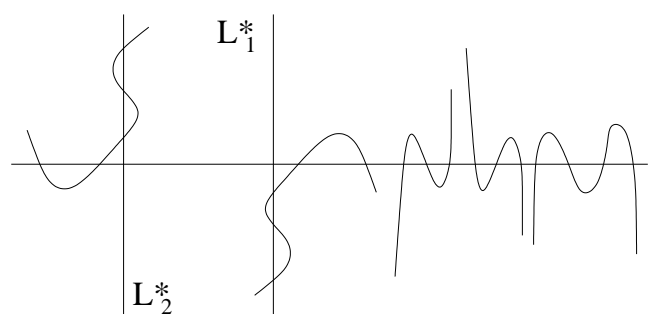

(even, $\left.A_{1}, A_{1}\right)$

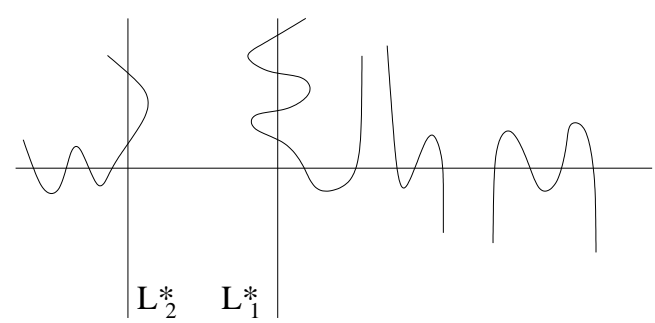

(odd, $\mathrm{A}_{0}, \mathrm{~A}_{0}$ )

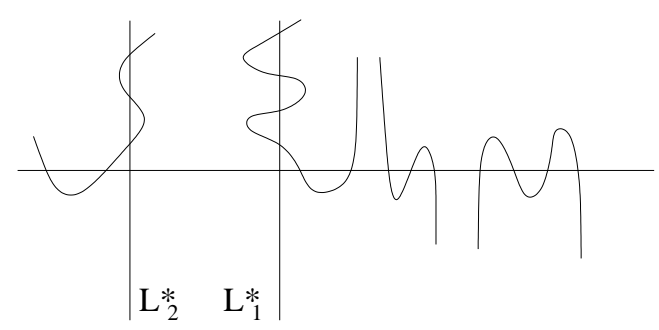

(odd, $\left.\mathrm{A}_{0}, \mathrm{~A}_{1}\right)$

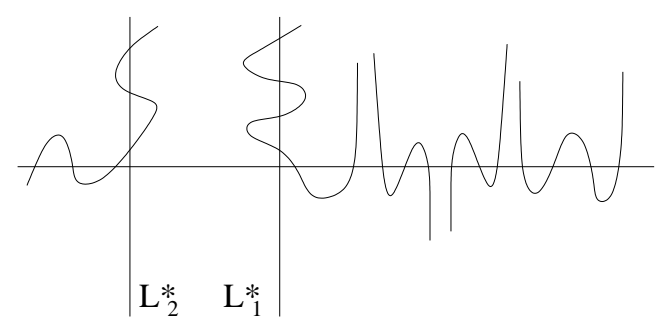

(odd, $\left.\mathrm{A}_{0}, \mathrm{~A}_{2}\right)$

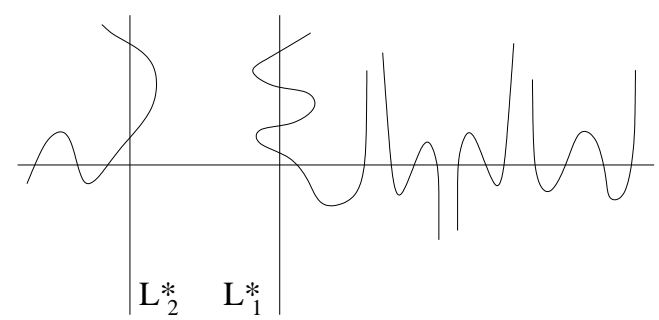

(odd, $\mathrm{A}_{0}, \mathrm{~A}_{3}$ )

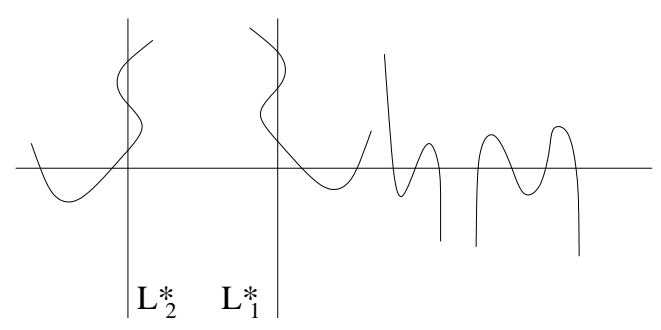

(odd, $\left.\mathrm{A}_{0}, \mathrm{~A}_{1}\right)$

Figure 7: Regular M-smoothings in the blown up plane I 


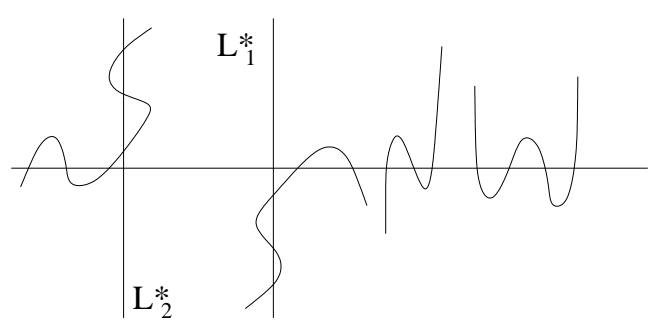

(even, $\mathrm{A}_{1}, \mathrm{~A}_{2}$ )

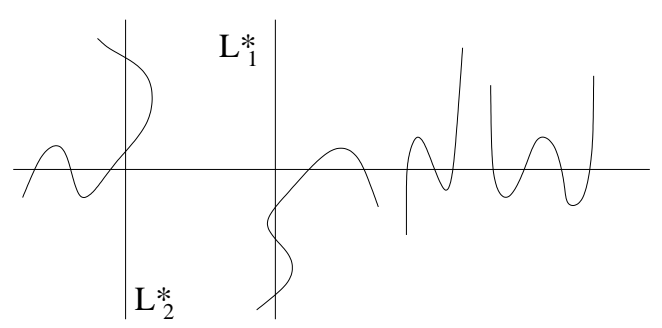

(even, $A_{1}, A_{3}$ )

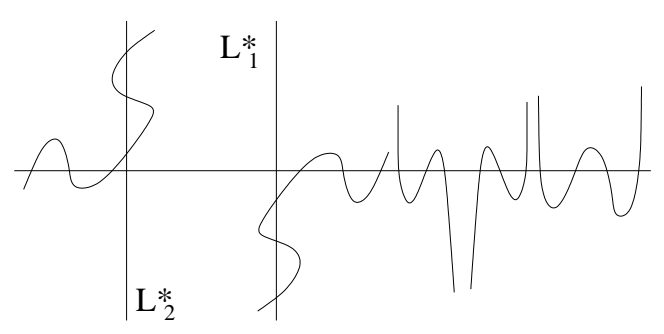

(even, $\mathrm{A}_{2}, \mathrm{~A}_{2}$ )

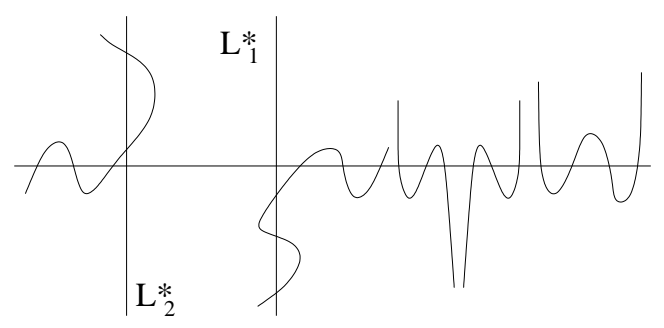

(even, $\mathrm{A}_{2}, \mathrm{~A}_{3}$ )

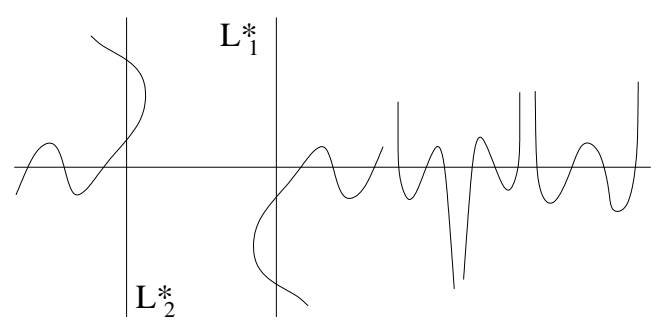

(even, $\mathrm{A}_{3}, \mathrm{~A}_{3}$ )

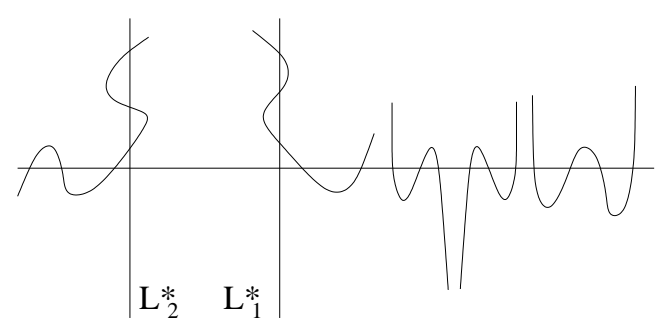

(odd, $\mathrm{A}_{1}, \mathrm{~A}_{2}$ )

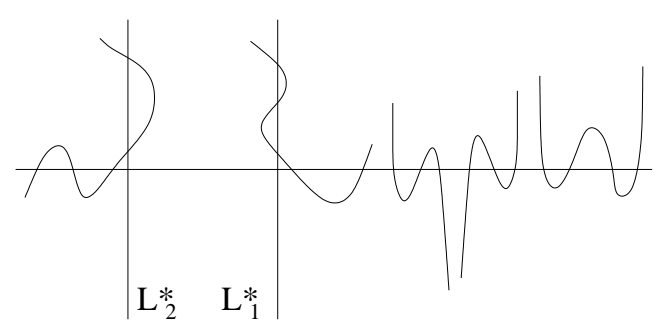

(odd, $\mathrm{A}_{1}, \mathrm{~A}_{3}$ )

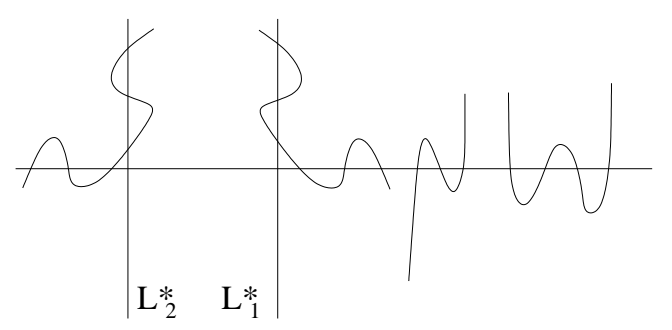

(odd, $\mathrm{A}_{2}, \mathrm{~A}_{2}$ )

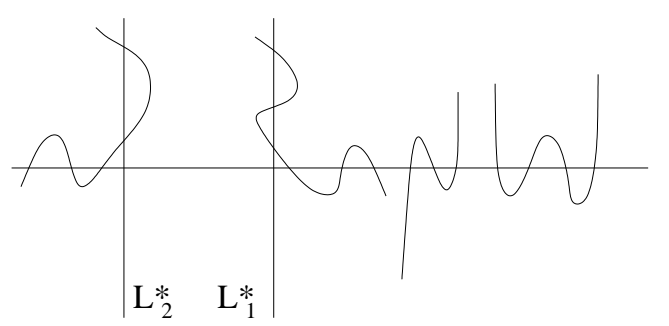

(odd, $\mathrm{A}_{2}, \mathrm{~A}_{3}$ )

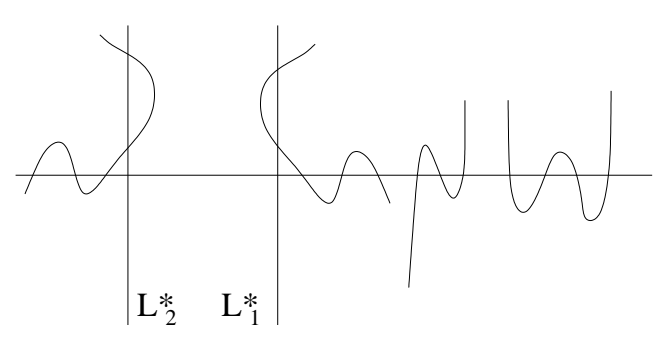

(odd, $\mathrm{A}_{3}, \mathrm{~A}_{3}$ )

Figure 8: Regular M-smoothings in the blown up plane II 


$$
(x, y) \in\left(\mathbb{R}^{*}\right)^{2} \mapsto \mu_{\Delta}(x, y)=\frac{\sum_{(i, j) \in \Delta \cap \mathbb{Z}^{2}}(i \cdot \operatorname{sign} x, j \cdot \operatorname{sign} y)|x|^{i}|y|^{j}}{\sum_{(i, j) \in \Delta \cap \mathbb{Z}^{2}}|x|^{i}|y|^{j}}
$$

it takes $\left(\mathbb{R}^{*}\right)^{2}$ diffeomorphically onto $\bigcup \operatorname{Int} \Delta_{\varepsilon, \delta}$. The chart ChF of $F$ is the closure of $\mu_{\Delta}\left(\{F=0\} \cap\left(\mathbb{R}^{*}\right)^{2}\right)$ (more precisely, the pair $(\Delta, \mathrm{Ch} F)$ ) considered up to homeomorphisms of $\Delta_{*}$ preserving each $\Delta_{\varepsilon, \delta}$ and its edges and vertices.

If $F$ and its truncations to the edges of $\Delta(F)$ have no singular points in $\left(\mathbb{R}^{*}\right)^{2}$, then $\mathrm{Ch} F$ is a topological curve with boundary properly embedded in $\Delta_{*}$. Polynomials satisfying the hypothesis of the above statement are called completely Newton nondegenerate (shortly CND).

Theorem 3 (see [22]) Let $F_{1}, \ldots, F_{N}$ be CND polynomials whose Newton polygons $\Delta_{1}, \ldots, \Delta_{N}$ have non empty interior and form a subdivision of a convex polygon $\Delta$. If all $F_{i}$ are the truncations of the same polynomial $\Phi=\sum_{(i, j) \in \Delta \cap \mathbb{Z}^{2}} A_{i j} x^{i} y^{j}$ and there is a convex piece-wise linear function $\nu: \Delta \rightarrow \mathbb{R}$ whose linearity domains are $\Delta_{1}, \ldots, \Delta_{N}$, then for any sufficiently small the polynomial $F(x, y)=$ $\sum_{(i, j) \in \Delta \cap \mathbb{Z}^{2}} A_{i j} t^{\nu(i, j)} x^{i} y^{j}$ is a CND polynomial and $\mathrm{Ch} V=\bigcup \mathrm{Ch} F_{i}$.

Remark 3 The function $\nu$ in theorem 3 can be corrected by a linear function in a way that on two prescribed neighboring facets $\sigma_{1}, \sigma_{2}$ of $\Delta$, or in some $\Delta_{k}$, the coefficients of $V$ will coincide with $A_{i j}$.

The patch-working construction from Theorem 3 feats into framework of toric varieties, see [22]. In particular, with any convex lattice polygon $\Delta$ is associated its toric surface $K \Delta$, which is a real algebraic surface obtained by some natural identification of edges of $\Delta_{*}$. This surface has at most isolated singular points, they correspond to a subset of vertices of $\Delta$. To each edge $\gamma$ of $\Delta$ there corresponds a real divisor $K \gamma$, which is topologically a circle. It intersects only the circles of the neighboring edges, and each one only at one point. These 2 points divide $K \gamma$ in 2 intervals $K \gamma_{ \pm}$. For any CND polynomial $F$ with Newton polygon $\Delta$ the image $K \mathrm{Ch} F$ of $\mathrm{Ch} F$ in $K \Delta$ is a closed topological 1-submanifold of the smooth part of $T \Delta$. The intersection points of $K \mathrm{Ch} F$ with $K \gamma_{+}$are the positive and with $K \gamma_{-}$the negative peripheral roots of $F$.

Under integral translations of $\Delta$ the pair $(K \Delta, K \mathrm{Ch} F)$ does not change, and under a $S L_{2}(\mathbb{Z})$ transformation of $\Delta$ it is replaced by a canonically homeomorphic pair and the canonical homeomorphism respects the stratification of $K \Delta$.

If, in notation of Theorem $3, K \mathrm{Ch} F$ is an $M$-curve (Harnack maximal), then $K \mathrm{Ch} F_{i}$ is an $M$-curve for any $i, 1 \leq i \leq N$. The inverse is not true. To remedy such a difficulty we introduce some natural regularity conditions.

A real quasihomogeneous polynomial is called steady, if it has no critical points in $\left(\mathbb{C}^{*}\right)^{2}$ and has no real peripheral roots of different signs (in particular, it can have only imaginary roots). A polynomial with Newton polygon $\Delta$ not reduced to an edge is called steady if it is a real CND polynomial with steady truncations to the edges of $\Delta$. 


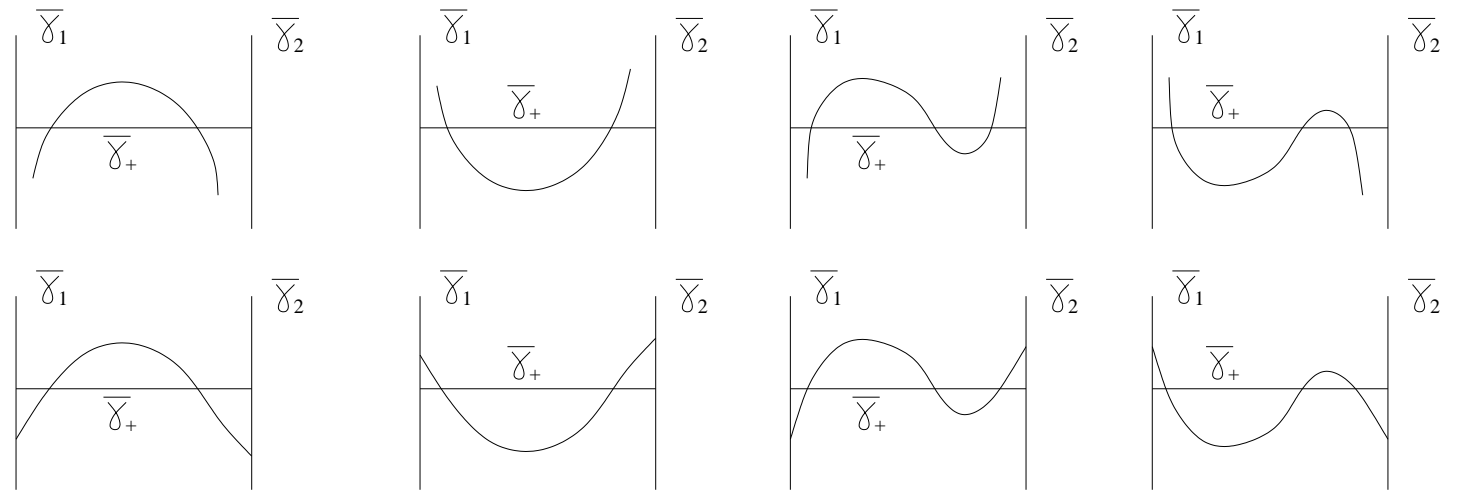

Figure 11: Weakly regular and regular intersections

Let $F$ be a real CND polynomial with $\operatorname{Int} \Delta(F) \neq \emptyset$. Suppose that $F^{\gamma}$ for some edge $\gamma$ of $\Delta$ is steady. Denote by $\gamma_{1}, \gamma_{2}$ the neighboring to $\gamma$ edges of $\Delta$. We speak of the weakly regular intersection of $F$ and $\gamma$, if there is a component of $K \mathrm{Ch} F \backslash \bigcup_{\gamma^{\prime} \neq \gamma} K \gamma^{\prime}$ which passes through all the points of $K \mathrm{Ch} F \cap K \gamma$ and these points are placed in $K \gamma$ in the same order as in this component. We call a weakly regular intersection of $F$ and $\gamma$ regular, if the above component continued joins the point of $K \mathrm{Ch} F \cap K \gamma$ most close to $\gamma_{1}$ with a point on $\gamma_{1}$ and the point of $K \mathrm{Ch} F \cap K \gamma$ most close to $\gamma_{2}$ with a point on $\gamma_{2}$ (see the lower part of Figure 11). If $F^{\gamma}$ has at least one (resp. two) real roots, then there are exactly two isotopy types of regular (resp, weakly regular) intersection of $F$ with $\gamma$ (see Figure 11).

Let $\gamma=\Delta_{1} \cap \Delta_{2}$ be a common edge of Newton polygons $\Delta_{1}, \Delta_{2}$ of polynomials $F_{1}, F_{2}$ with the same truncation on $\gamma$. Assume that both polygons have a nonempty interior. Regular, or weakly regular intersections of $F_{1}, F_{2}$ with $\gamma$ are called compatible, if $\mathrm{Ch} F_{1} \cup \mathrm{Ch} F_{2}$ contains $\#\left(\gamma \cap \mathbb{Z}^{2}\right)-2$ ovals intersecting $\gamma$ and its symmetric copies, and not intersecting the other edges (see Figure 12).

Theorem 3 is applied to patch-work smoothings of singularities. In particular, it has the following straightforward consequence.

Theorem 4 (see [22]) If in notation of Theorem 3, $\Delta_{N}$ intersects both the coordinate axes and $\Delta_{1}, \ldots, \Delta_{N-1}$ fill the domain $\mathcal{D}$ bounded by $\Delta_{N}$ and the coordinate axes, then the singular point $(C, 0)$, where $C$ is defined by $F_{N}=0$, has a smoothing $C^{\prime}$ with $\left(B_{\mathbb{R}}(C, 0), C_{\mathbb{R}}^{\prime}\right)$ homeomorphic to $\left(\mathcal{D}_{*}, \bigcup_{i=1}^{N-1} \mathrm{Ch} F_{i}\right)$.

$\left(\mathcal{D}_{*}\right.$ states for the union of the four mirror copies of $\mathcal{D}$.)

\subsection{Auxiliary M-polynomials with Newton triangles}

A CND polynomial $F$ is called $M$-polynomial if $\operatorname{Ch} F \cap\left(\mathbb{R}^{*}\right)^{2}$ has $\#\left(\mathbb{Z}^{2} \cap \operatorname{Int} \Delta(F)\right)+1$ ovals for the case when $F$ has no real peripheral roots, or has $\#\left(\mathbb{Z}^{2} \cap \operatorname{Int} \Delta(F)\right)$ ovals otherwise. Note that in the second case all noncompact components of $\operatorname{Ch} F \cap\left(\mathbb{R}^{*}\right)^{2}$ belong to one component of $K \mathrm{Ch} F$. 


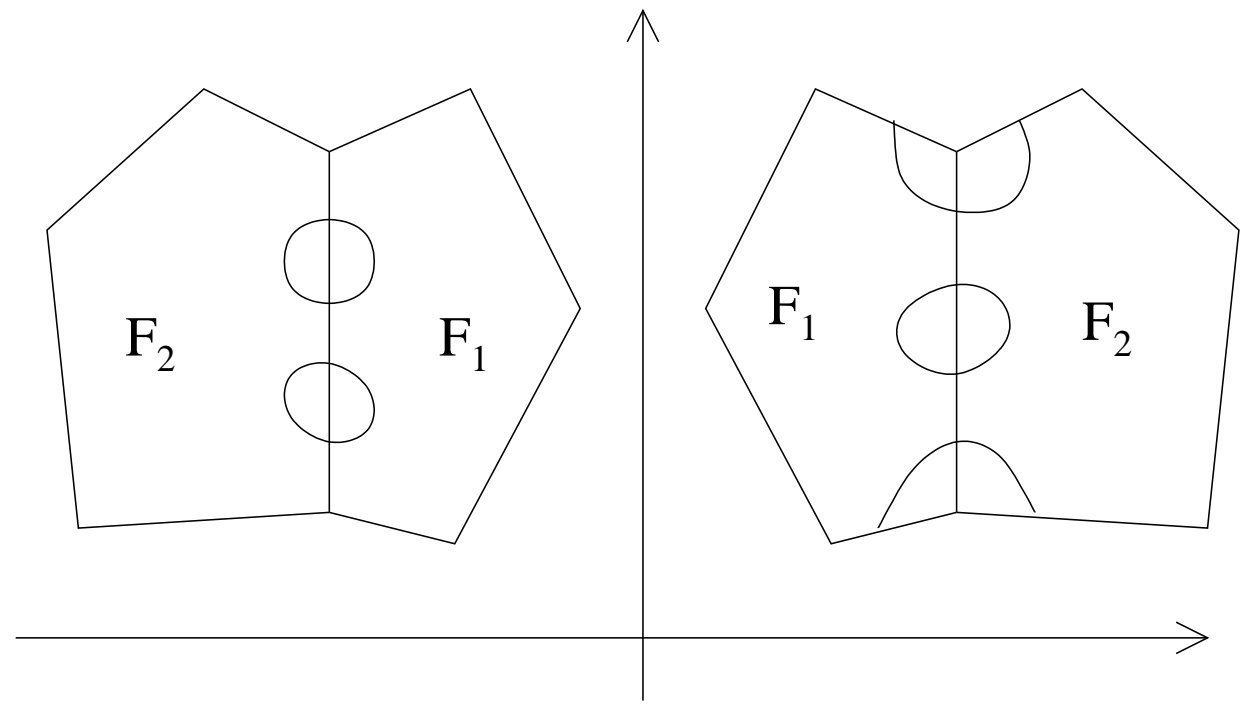

Figure 12: Compatible regular intersection

Proposition 4 Let $V_{1}, V_{2}$, and $V_{3}$ be integral points of $\mathbb{R}_{+}^{2}$ which form a nondegenerate triangle $T$ with edges $S_{1}=\left[V_{2}, V_{3}\right], S_{2}=\left[V_{1}, V_{3}\right]$, and $S_{3}=\left[V_{1}, V_{2}\right]$, and let $h$ be a steady quasihomogeneous polynomial with $\Delta(h)=S_{1}$ and with at least one real peripheral root. Then there exists an $M$-polynomial $F$ with $\Delta(F)=T$ such that: $h=F^{S_{1}} ; F^{S_{2}}$ and $F^{S_{3}}$ are steady and have only real peripheral roots; the intersections of $F$ with $S_{1}, S_{2}$, and $S_{3}$ are regular, and the isotopy type of the regular intersection with $S_{1}$ can be prescribed.

Proof. We proceed by induction on the number $s$ of integral points inside $T$. If $s=0$, a suitable $S L_{2}(\mathbb{Z})$ transformation combined with a translation takes $T$ into a triangle with vertices $(0,0),(0,1),(k, 0)$, or $(0,0),(2,0),(0,2)$. For such a triangle the statement is obvious.

Assume that $s>0$. Take the closest to $S_{1}$ integral point $V \in \operatorname{Int} T$ and divide $T$ into the triangles

$$
\tau_{1}=\operatorname{conv}\left\{V, V_{2}, V_{3}\right\}, \quad \tau_{2}=\operatorname{conv}\left\{V, V_{1}, V_{3}\right\}, \quad \tau_{3}=\operatorname{conv}\left\{V, V_{1}, V_{2}\right\}
$$

(conv states for the convex hull). Inside each of $\tau_{1}, \tau_{2}, \tau_{3}$ there are less than $s$ integral points, and we can apply the induction assumption. First, take a steady M-polynomial $F_{1}$ with: $\Delta\left(F_{1}\right)=\tau_{1}, F_{1}^{S_{1}}=h$ and the isotopy type of regular intersection with $S_{1}$ which is prescribed for $F$. Thus, in a prescribed quadrant $\mathbb{R}_{\varepsilon}^{2}$ the components of $\mathrm{Ch} F$ intersecting $S_{1}$ are placed as shown in Figure 13a. In addition, $\mathrm{Ch} F_{1}$ has in some other quadrant, say, in the positive one, an arc connecting the edges $\left[V, V_{2}\right],\left[V, V_{3}\right]$ (see Figure $13 \mathrm{a}$ ). Then, pick a steady quasihomogeneous polynomial $h^{\prime}$ with: $\Delta\left(h^{\prime}\right)=\left[V, V_{1}\right]$, all peripheral roots real and the same coefficient at $V$ as in $F_{1}$. By induction assumption, there exist steady M-polynomials $F_{2}, F_{3}$ with $\Delta\left(F_{2}\right)=\tau_{2}, \Delta\left(F_{3}\right)=\tau_{3}$ and $F_{2}^{\left[V, V_{1}\right]}=F_{3}^{\left[V, V_{1}\right]}=h^{\prime}$, and which have a compatible 


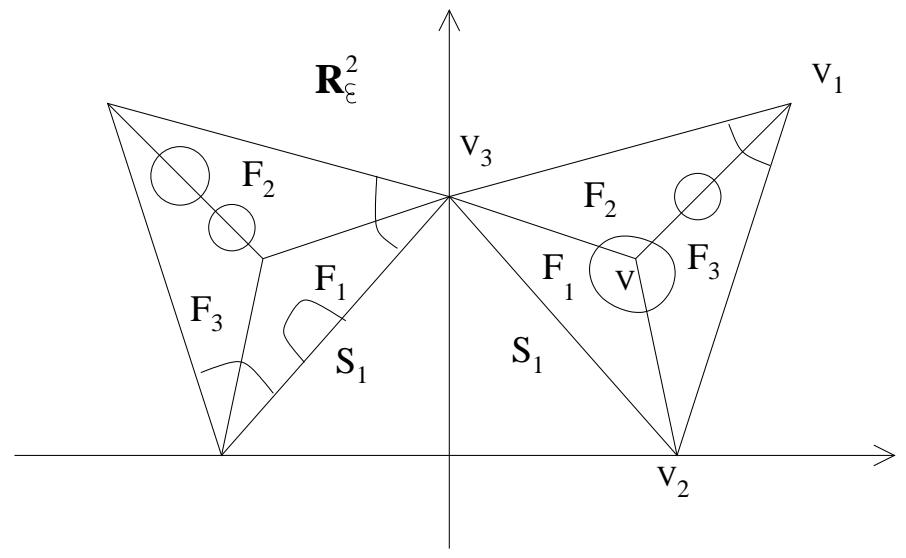

Figure 13: M-polynomials with Newton triangles

regular intersection with $\left[V, V_{1}\right]$ such that $\mathrm{Ch} F_{2}$ (resp. $\left.\mathrm{Ch} F_{3}\right)$ has in $\tau_{2}$ (resp. $\tau_{3}$ ) an arc connecting the edges $\left[V, V_{1}\right],\left[V, V_{3}\right]$ (resp. $\left.\left[V, V_{1}\right],\left[V, V_{2}\right]\right)$. By construction, $V, V_{2}, V_{3}$ are the only integral points on the edges $\left[V, V_{2}\right],\left[V, V_{3}\right]$, and the sign of the coefficient of $F_{1}, F_{2}\left(\operatorname{resp} . F_{1}, F_{3}\right)$ at $V_{3}\left(\operatorname{resp} . V_{2}\right)$ is the same. Hence we can equalize the coefficients of $F_{1}, F_{2}$ (resp. $\left.F_{1}, F_{3}\right)$ at $V_{3}$ (resp. $V_{2}$ ) by a suitable transformation

$$
F_{2}(x, y) \mapsto \lambda_{0} F_{2}\left(\lambda_{1} x, \lambda_{2} y\right), \quad F_{3}(x, y) \mapsto \lambda_{0}^{\prime} F_{2}\left(\lambda_{1}^{\prime} x, \lambda_{2}^{\prime} y\right), \quad \lambda_{i}, \lambda_{i}^{\prime}>0, i=0,1,2,
$$

keeping the truncation $h^{\prime}$ on $\left[V, V_{1}\right]$. The isotopy type of regular intersection of $F_{2}$ and $F_{3}$ with this edge is preserved by such transformations.

Now we apply Theorem 3 and Remark 3 to $F_{1}, F_{2}, F_{3}$ and get a polynomial $V$ with $V_{1}^{S}=h$ : the convex function $\nu$ can be constructed by a perturbation of a linear one. The polynomial $V$ thus obtained is an M-polynomial, since $\mathrm{Ch} V$ has: $\sum \#\left(\operatorname{Int}\left(\tau_{i}\right) \cap \mathbb{Z}^{2}\right)$ ovals coming from $\bigcup\left(\mathrm{Ch} F_{i} \cap\left(\mathbb{R}^{*}\right)^{2}\right) ; \#\left(\left[V, V_{1}\right] \cap \mathbb{Z}^{2}\right)-2$ ovals coming from gluing $\mathrm{Ch} F_{2}$ and $\mathrm{Ch}_{3}$ along $\left[V, V_{1}\right]$ and its symmetric copies; and one more oval appearing around the point $V$ (see Figure 13a), which gives a total of

$$
\sum \#\left(\operatorname{Int}\left(\tau_{i}\right) \cap \mathbb{Z}^{2}\right)+\#\left(\left[V, V_{1}\right] \cap \mathbb{Z}^{2}\right)-2+1=\#\left(\operatorname{Int}(T) \cap \mathbb{Z}^{2}\right)
$$

ovals.

Further, $F$ has regular intersections with $S_{1}, S_{2}, S_{3}$ and the type of the regular intersection with $S_{1}$ is as prescribed. Indeed, $\mathbb{R}_{\varepsilon}^{2}$ is not the positive quadrant (see above), and, thus, the only arc of $\mathrm{Ch}_{2} \cap \mathbb{R}_{\varepsilon}^{2}$ with the endpoint on $\left[V, V_{3}\right]$ does not go to the edge $\left[V, V_{1}\right]$ (the intersection of $F_{2}$ with this edge is regular); hence, it goes to the edge $S_{2}$, completing the prescribed regular intersection of $F$ with $S_{1}$.

Proposition 5 Let $T \subset \mathbb{R}^{2}$ be a triangle whose vertices $V_{1}, V_{2}, V_{3}$ have nonnegative integral coordinates and the edges $S_{2}=\left[V_{1}, V_{3}\right], S_{3}=\left[V_{1}, V_{2}\right]$ contain no integral points except for $V_{1}, V_{2}, V_{3}$. If $h$ is a steady quasihomogeneous polynomial with $\Delta(h)=\left[V_{2}, V_{3}\right]$ and without real peripheral roots, then there exists an M-polynomial $F$ with $\Delta(F)=T$ and $h=F^{S_{1}}$, where $S_{1}=\left[V_{2}, V_{3}\right]$. 


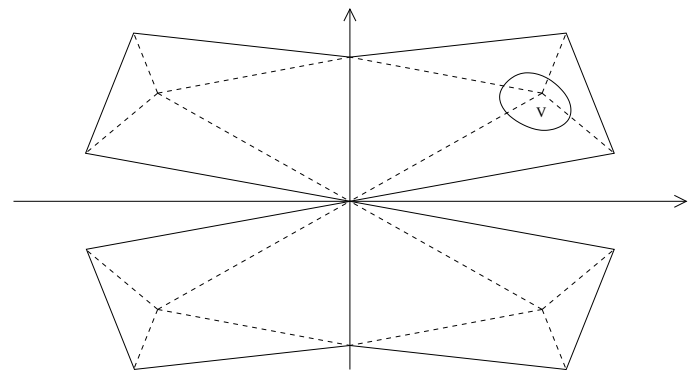

a)

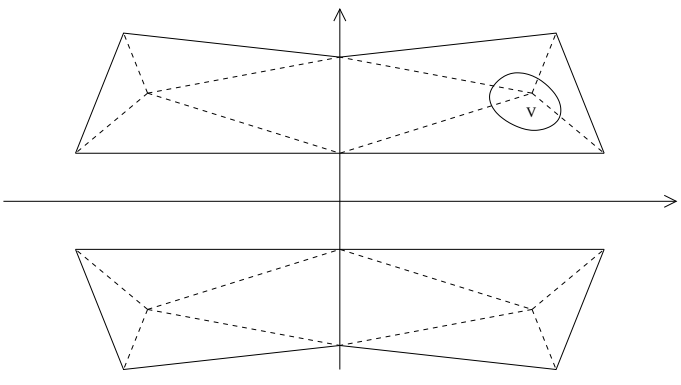

b)

Figure 15: M-polynomials with Newton quadrangles I

coordinate axes into triangles as shown in Figure 14 (the existence of an appropriate convex function $\nu$ follows from the convexity of the Newton diagram) and then patchwork suitable M-polynomials from Propositions 4, 5 with these Newton triangles and given truncations on the edges on the Newton diagram (note that the edges inside the diagram are without integral points in their interior and recall that $\frac{1}{2}(\mu(C, z)-$ $r(C, z)+1)$ is equal to the number of integral points in the interior of the domain bounded by the coordinate axes and the Newton diagram). For instance, in the case when the singularity is given by equation (3) with $a=b=0$ and $c s, d s$ both even, if the peripheral roots are all imaginary we use the subdivision into three triangles shown in Figure 14a (in each triangle two edges have no integral points in their interior) and patch-work three M-polynomials given by Proposition 5. The result provides an M-smoothing with $\frac{1}{2}(\mu(C, z)-r(C, z)+3)$ ovals: namely, $\frac{1}{2}(\mu(C, z)-$ $r(C, z)+1)-1$ ovals come from the ovals in $\left(\mathbb{R}^{*}\right)^{2}$ of the polynomial with Newton triangle $\operatorname{conv}\{(1,1),(0, c s),(d s, 0)\}$, one more oval appears around the point $(1,1)$, and one more around $(-1,-1)$.

\subsection{Auxiliary M-polynomials with Newton quadrangles}

Let $p, q, s$ be positive integers. Consider the polygons $Q_{p q s}^{\prime}=\operatorname{conv}\{A, B, C, D\}$ and $Q_{p q s}^{\prime \prime}=\operatorname{conv}\left\{A^{\prime}, B, C, D\right\}$ where $A=(0,0), B=(0, p), C=(q, p+1), D=(q+s, 1)$, and $A^{\prime}=(0,1)$. Note that the edges $[A D]$ and $[B C]$ contain no integral point in their interior, and, thus, real polynomials corresponding to them are all steady, each of them has only one peripheral root and this root is real.

Proposition 7 Let $h_{1}$ and $h_{2}$ be steady quasi-homogeneous polynomials with $\Delta\left(h_{1}\right)=[B C]$ and $\Delta\left(h_{2}\right)=[C D]$. Assume that $p$ and $s$ are even, $h_{1}$ and $h_{2}$ have the same coefficient of $x^{q} y^{p+1}$, and $h_{2}$ has no real peripheral roots. Then there exist M-polynomials $F^{\prime}$ and $F^{\prime \prime}$ with $\Delta\left(F^{\prime}\right)=Q_{p q s}^{\prime}, \Delta\left(F^{\prime \prime}\right)=Q_{p q s}^{\prime \prime},\left(F^{\prime}\right)^{[B C]}=h_{1}$, and $\left(F^{\prime \prime}\right)^{[C D]}=h_{2}$.

Proof. Take the closest to $[C D]$ integral point $V$ of $\operatorname{Int}\left(Q_{p q s}^{\prime}\right)$ and divide $Q_{p q s}^{\prime}$ into four triangles joining $V$ with the vertices of $Q_{p q s}^{\prime}$ (see Figure 15a): $\tau_{1}=\operatorname{conv}\{B, C, V\}, \tau_{2}=\operatorname{conv}\{A, B, V\}, \tau_{3}=\operatorname{conv}\{C, D, V\}, \tau_{4}=\operatorname{conv}\{A, D, V\}$. 
Let $F_{1}$ be an M-polynomial with $\Delta\left(F_{1}\right)=\tau_{1}$ and $\left(F_{1}{ }^{[B C]}=h_{1}\right.$ given by Proposition 4. Its chart has an arc connecting $[B C]$ and $[C D]$ in $\tau_{1}$ or in one of the symmetric copies of $\tau_{1}$; without lost of generality, we may suppose that it happens in $\tau_{1}$. Proposition 4 provides an M-polynomial $F_{2}$ with $\Delta\left(F_{2}\right)=\tau_{2},\left(F_{2}\right)^{[B V]}=\left(F_{1}\right)^{[B V]}$, and such that $F_{1}$ and $F_{2}$ have a compatible regular intersection with $[B V]$. In particular, $\mathrm{Ch}\left(F_{2}\right)$ has in $\tau_{2}$ an arc connecting $[B V]$ and $[A V]$. By Proposition 5 there exists an M-polynomial $F_{3}$ with $\Delta\left(F_{3}\right)=\tau_{3},\left(F_{3}\right)^{[C D]}=h_{2}$, and $\operatorname{Ch}\left(F_{3}\right)$ joining $[C V]$ and $[D V]$ in $\tau_{3}$ by an arc.

By construction, $[C V]$ has the endpoints as its only integral points, the coefficients at the monomial $x^{q} y^{p+1}$ corresponding to $\mathrm{C}$ in $F_{1}$ and $F_{3}$ coincide, and the coefficients at the monomial $x^{u} y^{v}$ corresponding to $V$ in $F_{1}$ and $F_{3}$ have the same sign. One can equalize the latter coefficients by a suitable transformation (4) applied to $F_{3}$, preserving all the properties of $F_{3}$ cited above. Finally, Proposition 4 provides an Mpolynomial $F_{4}$ with $\Delta\left(F_{4}\right)=\tau_{4},\left(F_{4}\right)^{[A V]}=\left(F_{2}\right)^{[A V]}$, and such that $F_{4}$ and $F_{2}$ have a compatible intersection with $[a v]$. In particular, $\mathrm{Ch}\left(F_{3}\right)$ has in $\tau_{4}$ an arc joining $[A V]$ and $[D V]$. It implies that the coefficients at the monomial $x^{q+s} y$ corresponding to $D$ in $F_{3}$ and $F_{4}$ have the same sign, and to equalize the truncations of $F_{3}$ and $F_{4}$ onto $[D V]$, one has only to correct the absolute value of this coefficient in $F_{4}$, which can be made as in (4).

Now, it remains to apply Theorem 3 and patch-work $F_{1}, F_{2}, F_{3}$, and $F_{4}$ (a suitable convex function $\nu$ is obtained by a small variation of a linear function). The result is an M-polynomial, since its chart has: $\bigcup_{i=1}^{4} \#\left(\operatorname{Int}\left(\tau_{i}\right) \cap \mathbb{Z}^{2}\right)$ ovals which come from the ovals of the glued charts, \#((Int $\left.[A V] \cup \operatorname{Int}[B V]) \cap \mathbb{Z}^{2}\right)$ ovals which come from gluing compatible intersections along $[A V]$ and $[B V]$ and their symmetric images, and one more oval around $V$.

The polynomial $F^{\prime \prime}$ is constructed in the same way (see Figure $15 \mathrm{~b}$ ).

Proposition 8 Let $h_{1}$ and $h_{2}$ be steady quasi-homogeneous polynomials with $\Delta\left(h_{1}\right)=[B C]$ and $\Delta\left(h_{2}\right)=[C D]$. Assume that $p, s$ are coprime and $h_{1}, h_{2}$ have the same coefficient of $x^{q} y^{p+1}$. If $p$ is odd and $q, s$ are even, assume additionally that the peripheral roots of $h_{1}, h_{2}$ have the same sign. Then there exist M-polynomials $F^{\prime}$ and $F^{\prime \prime}$ with $\Delta\left(F^{\prime}\right)=Q_{p q s}^{\prime}, \Delta\left(F^{\prime \prime}\right)=Q_{p q s}^{\prime \prime},\left(F^{\prime}\right)^{[B C]}=h_{1}$, and $\left(F^{\prime \prime}\right)^{[C D]}=h_{2}$.

Proof. Assume that $B, C$, and $D$ are not collinear $\bmod 2$, i.e.,

$$
p q-s \equiv 1 \quad \bmod 2 .
$$

Divide $Q_{p q s}^{\prime}$ into two triangles: $\tau_{1}=\operatorname{conv}\{B, C, D\}$ and $\tau_{2}=\operatorname{conv}\{A, B, D\}$ (see Figure 16a). By Proposition 4 there exists an M-polynomial $F_{1}$ with $\Delta\left(F_{1}\right)=\tau_{1}$, $F_{1}^{[B C]}=h_{1}$ and regular intersection with $[B D]$. Since the vertices of $\tau_{1}$ are not collinear mod 2, suitable transformations (4) followed by $F_{1}(x, y) \mapsto \pm F_{1}( \pm x, \pm y)$ makes the coefficient at $x^{q+s} y$ corresponding to $D$ in $F_{1}$ equal to that in $h_{2}$, keeping the truncation to $[B C]$. Complete the construction of $F^{\prime}$, patch-working $F_{1}$ with an M-polynomial $F_{2}$ which has $\Delta\left(F_{2}\right)=\tau_{2}$ and $\left.F_{2}^{[B D]}=F_{1}^{[B D}\right]$, and whose chart intersects $[B D]$ compatibly with $\mathrm{Ch}\left(F_{1}\right)$. 


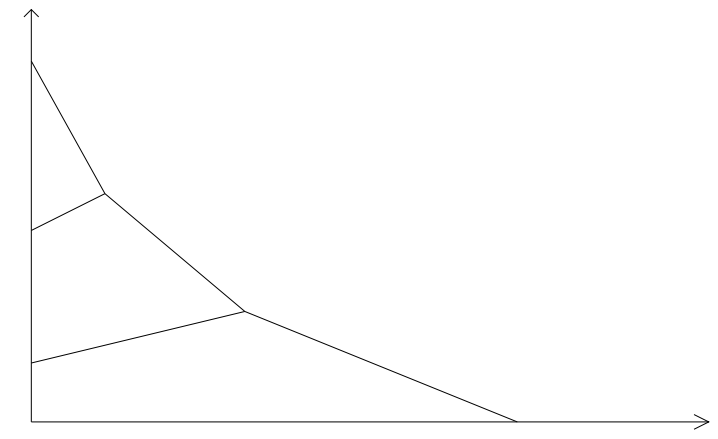

Figure 17: M-smoothing of isolated Newton nondegenerate singular point

\subsection{Proof of Theorem 2(2)}

We assume that $c, d$ in (3) describing the leading part $f^{\gamma}$ of the given real semiquasi-homogeneous singularity satisfy:

- $c, d>1$, since otherwise, say, for $c=1$ a transformation $y \mapsto y-A x^{d}$ makes all the real peripheral roots of $f^{\gamma}$ positive and, hence, reduces the problem to Theorem 2(1);

- $d$ is odd, since $c, d$ are coprime and we can interchange $x, y$ if necessary.

Assume also that $f^{\gamma}$ has $2 k_{0}$ imaginary, $k_{+}>0$ positive and $k_{-}>0$ negative peripheral roots (otherwise, Theorem 2(2) follows from Theorem 2(1)). Trace a segment $[(i, j),(0, j-1)]$ for any integral point $(i, j) \in \gamma, i, j>0$. We obtain a polygon $\Delta$ which is either a triangle (case $a=0$ ) or a quadrangle (case $a=1$ ), has $\gamma$ as an edge, and is subdivided in polygons (also triangles and quadrangles) $\Delta_{i}$, $i=1, \ldots, k=2 k_{0}+k_{+}+k_{-}$, which we number from up downwards. Put

$$
Q_{0}=\Delta_{1} \cup \ldots \cup \Delta_{2 k_{0}}, \quad Q_{i}=\Delta_{2 k_{0}+i}, i=1, \ldots, k_{+}+k_{-} .
$$

Pick: a polynomial $h_{0}$ with $\Delta\left(h_{0}\right)=\gamma \cap Q_{0}$ which has $2 k_{0}$ distinct imaginary peripheral roots; polynomials $h_{i}, i=1, \ldots, k_{+}$, with $\Delta\left(h_{i}\right)=\gamma \cap Q_{i}$ which have positive peripheral roots; and polynomials $h_{i}, i=k_{+}+1, \ldots, k_{+}+k_{-}$, with $\Delta\left(h_{i}\right)=$ $\gamma \cap Q_{i}$ which have negative peripheral roots. Note that up to vertical shifts: $Q_{i}$, $i=1, \ldots, k_{+}+k_{-}-1$, are quadrangles of type $Q_{p q s}^{\prime} ; Q_{0}$ is a triangle as in Proposition 6 or a quadrangle of type $Q_{p q s}^{\prime}$, according as $a=0$ or 1 in (3); and $Q_{k_{+}+k_{-}}$is a quadrangle of type $Q_{p q s}^{\prime}$ or $Q_{p q s}^{\prime \prime}$, according as $b=1$ or 0 in (3). Note also that all these polygons satisfy the corresponding conditions of Propositions $6,7,8$, and the case which is not covered by Proposition 8 (i.e. $p$ is odd, $q, s$ are even, the roots of $h_{1}, h_{2}$ have different signs) never occurs because of initial adjustment of $c$ and $d$.

Take an M-polynomial with $\Delta\left(F_{0}\right)=Q_{0}$ and $F_{0}^{\gamma \cap Q_{0}}=h_{0}$ which satisfies the conditions of Proposition 6 or 7 , according as $Q_{0}$ is triangle or quadrangle. By induction, construct M-polynomials $F_{i}, i=1, \ldots, k_{+}+k_{-}$, with $\Delta\left(F_{i}\right)=Q_{i}$ and $F_{i}^{\gamma \cap Q_{i}}=h_{i}$ satisfying the conditions of Proposition 8 so that $F_{i}$ truncated on the upper edge of $Q_{i}$ coincides with $F_{i-1}$ truncated on the lower edge of $Q_{i-1}$. 
Theorem 3 applied to $F_{i}$ as above gives us an M-polynomial $F$ with $\Delta(F)=\Delta$ : a suitable function $\nu$ is constructed by induction, and $F$ is an $M$-polynomial since

$$
\sum_{i=0}^{k_{+}+k_{-}} \#\left(\operatorname{Int} Q_{i} \cap \mathbb{Z}^{2}\right)=\#\left(\operatorname{Int} \Delta \cap \mathbb{Z}^{2}\right) .
$$

The truncation $\tilde{f}=F^{\gamma \cap Q}$ is a quasi-homogeneous polynomial obtained by patchworking polynomials $h_{i}$. Thus, $\widetilde{f}$ is Newton nondegenerate and it has the same numbers of positive, negative and imaginary peripheral roots as $f^{\gamma}$. Therefore, $\widetilde{f}=0$ defines at the origin a singular point deformation equivalent over $\mathbb{R}$ to $f=0$. It remains to observe that $F=0$ can be considered as an M-smoothing of $\widetilde{f}=0$ : a smoothing family is given by $F_{t}(x, y)=t^{u} F\left(x t^{-c}, y t^{-d}\right), u=\left(2 k_{0}+k_{+}+k_{-}\right) c d+$ $a c+b d$, and it is an M-smoothing due to (2) and (6).

Remark 4 The end of the proof can be replaced by reference to Theorem 4 . If $a=1$ or $b=1$, then, first, $\Delta$ should be extended by one or two triangles, as well as by the convex envelope of the Newton diagram, to fill completely the domain between the diagram and the coordinate axes. The deformation type over $\mathbb{R}$ of the singularity remains unchanged.

\section{Weak M-smoothing of Newton nondegenerate singular points}

The local Harnack inequality (1) can be refined in the following way (see, for instance, [8]). Given a real isolated singular point $(C, z)$, the intersection of $C$ with the circle $\partial B(C, z)_{\mathbb{R}}$ is decomposed in pairs of points belonging to one branch of $C$. Given a real smoothing $C^{\prime}$ of $(C, z)$, this decomposition is transported to the intersection of $C^{\prime}$ with $\partial B(C, z)_{\mathbb{R}}$ : two boundary points of $C_{\mathbb{R}}^{\prime}$ belong to the same pair, and called boundary equivalent, if they belong to the same component of $C^{\prime} \cap \partial B(C, z)$. The refined inequality states that

$$
v \leq \frac{\mu-r+3}{2}-l
$$

where $l$ is the number of topological circles obtained from the union of nonclosed components of $C_{\mathbb{R}}^{\prime}$ by identification of boundary equivalent points. A smoothing $C^{\prime}$ is called a weak $M$-smoothing if it provides an equality in (7).

The Harnack inequality for CND polynomials is refined in a similar way. Given a real CND polynomial $F$ in 2 variables, call two ends of $\{F=0\} \cap\left(\mathbb{R}^{*}\right)^{2}=$ $\mathrm{Ch}(F) \cap(\operatorname{Int} \Delta(F))_{*}$ (as usually, ()$_{*}$ stands for the union of four mirror images) equivalent if they merge into the same end of $\{F=0\} \cap\left(\mathbb{C}^{*}\right)^{2}$, i.e., define the same point on $K \Delta$. The refined inequality states that

$$
v \leq \#\left(\operatorname{Int} \Delta(F) \cap \mathbb{Z}^{2}\right)-l+1
$$


where $v$ is the number of ovals of $\{F=0\} \cap\left(\mathbb{R}^{*}\right)^{2}$ and $l$ is the number of topological circles obtained from the union of nonclosed components of $\{F=0\} \cap\left(\mathbb{R}^{*}\right)^{2}$ by identification of equivalent ends. A real CND polynomial is called a weak $M$-polynomial if it provides an equality in (8). (For related information see section 6 .)

The following simple lemma and its corollary are well known (see, [10]).

Lemma 9 A smoothing $C^{\prime}$ (respectively, a real $C N D$ polynomial $F$ ) is a weak $M$ smoothing (respectively, a weak M-polynomial) if, and only if, $C_{\mathbb{C}}^{\prime} \backslash C_{\mathbb{R}}^{\prime}$ (respectively, $\left.\{F=0\} \cap\left(\mathbb{C}^{*}\right)^{2} \backslash\{F=0\} \cap\left(\mathbb{R}^{*}\right)^{2}\right)$ is the disjoint union of two spheres with holes.

Corollary 9 If in notation of Theorem 3 (respectively, Theorem 4) $F_{1}, \ldots, F_{N}$ (respectively, $\left.F_{1}, \ldots, F_{N-1}\right)$ are weak $M$-polynomials, the adjacency graph of the decomposition $\Delta=\Delta_{1} \cup \cdots \cup \Delta_{N}$ (respectively, $\Delta_{1} \cup \cdots \cup \Delta_{N-1}$ ) is a tree, and the adjacency edges $\Delta_{i} \cap \Delta_{j}$ are without internal integral points, then $F$ is a weak M-polynomial (respectively, weak M-smoothing).

Theorem 5 Any Newton nondegenerate singular point is deformation equivalent over $\mathbb{R}$ to a Newton nondegenerate singular point which has a weak M-smoothing.

Remark 5 The number $l$ in (7) is 0 if $r_{\mathbb{R}}=0$ and $\geq 1$ if $r_{\mathbb{R}} \geq 1$. For the weak M-smoothings which we construct in the proof of Theorem 5 it can be estimated as follows. Call a real branch of a singular point a $(0,1)$-branch (or $(1,0)$ branch) if its intersection number with a real line through the singular point is even (resp. odd) if the line is tangent and odd (resp. even) otherwise. Then, $l \leq 1+\min \{\#((0,1)$-branches $), \#((1,0)$-branches $)\}$. Note also that a weak Msmoothing with $l=1$ is an M-smoothing.

In the proof of Theorem 5 we use the following auxiliary polynomials.

Proposition 10 Let $p, g, s$ be positive integers and $h_{1}, h_{2}$ polynomials with $\Delta\left(h_{1}\right)=$ $[B C]$ and $\Delta\left(h_{2}\right)=[C D]$, where $B=(0, p), C=(q, p+1)$, and $D=(q+s, 1)$. Assume that $p$ and $s$ are coprime, $q$ and $s$ are even, $p$ is odd, the (only) peripheral root of $h_{1}$ is positive, and the (only) peripheral root of $h_{2}$ is negative.

(1) There exists a weak M-polynomial $F$ with $\Delta(F)=Q_{p q s}^{\prime}, Q_{p q s}^{\prime}=$ $\operatorname{conv}(A, B, C, D), A=(0,0)$, and such that: $F^{[B C]}=h_{1}, F^{[C D]}=h_{2}, \mathrm{Ch} F$ has $\#\left(\operatorname{Int}\left(Q_{p q s}^{\prime}\right) \cap \mathbb{Z}^{2}\right)-1$ ovals and a connected component of $\operatorname{Ch}(F)$ joins $[B C]$ and $B \bar{C}, \bar{C}=(-q, p+1)$, as shown in Figure $18 a$.

(2) If $p>1$, then there exists a weak M-polynomial $F$ with $\Delta(F)=Q_{p q s}^{\prime \prime}, Q_{p q s}^{\prime \prime}=$ $\operatorname{conv}\left(A^{\prime}, B, C, D\right), A^{\prime}=(0,1)$, and such that: $F^{[B C]}=h_{1}, F^{[C D]}=h_{2}$, ChF has $\#\left(\operatorname{Int}\left(Q_{\text {pqs }}^{\prime \prime}\right) \cap \mathbb{Z}^{2}\right)-1$ ovals and a connected component of $\operatorname{Ch}(F)$ joins $[B C]$ and $B \bar{C}, \bar{C}=(-q, p+1)$, as shown in Figure $18 b$.

(3) The same statements (1) and (2) hold true if the root of $h_{1}$ is negative, the root of $h_{2}$ is positive, and the above charts are replaced by their symmetric images with respect to the horizontal axis. 
Examining the sequence of polygons $Q_{u}^{(i)}$ from up downwards, construct a sequence of polynomials $F_{u}^{(i)}$ with $\Delta\left(F_{u}^{(i)}\right)=Q_{u}^{(i)}$ such that: their truncations to the edges $Q_{u}^{(i)} \cap \gamma_{u}$ are $h_{u}^{(i)}$, their truncations to adjacent edges of neighboring polygons coincide, and each $F_{u}^{(i)}$ satisfies the conditions of Propositions 4, 6, 7, 8, 10, respectively.

For each $u=1, \ldots, n$ apply Theorem 3 to patch-work $F_{u}^{(i)}, i=0, \ldots, k_{u}^{+}+k_{u}^{-}$, into a polynomial $F_{u}$ with $\Delta\left(F_{u}\right)=Q_{u}=\bigcup_{i} Q_{u}^{(i)}$ (a suitable convex function $\nu$ is constructed by induction). The truncations of $F_{u}$ and $F_{u+1}$ on $\sigma=[(i, j),(0, j-1)]=$ $Q_{u} \cap Q_{u+1}$ are

$$
F_{u}^{\sigma}=t_{1} A y^{j-1}+t_{2} B x^{i} y^{j}, \quad F_{u+1}^{\sigma}=t_{3} A y^{j-1}+t_{4} B x^{i} y^{j}, \quad t_{1}, t_{2}, t_{3}, t_{4}>0 .
$$

Equalize them successively applying the transformation (4) to $F_{2}, \ldots, F_{n}$, and keeping the signs of the peripheral roots of $F_{u}^{\gamma_{u}}, u=2, \ldots, n$.

Let $\tilde{f}$ be a polynomial such that $\gamma_{1} \cup \ldots \cup \gamma_{n}$ is a part of the boundary of its Newton polygon, and $\widetilde{f}^{\gamma_{u}}=F_{u}^{\gamma_{u}}, u=1, \ldots, n$. By construction, $\widetilde{f}$ and $f$ has the same numbers of imaginary, positive and negative peripheral roots on each edge $\gamma_{u}$. Therefore, $\widetilde{f}$ defines a singular point $(\widetilde{C}, 0)$ deformation equivalent over $\mathbb{R}$ to $(C, 0)$. To obtain a smoothing $\widetilde{C}^{\prime}$ of $(\widetilde{C}, 0)$ it remains to apply Theorem 4 to $F_{u}, 1 \leq u \leq n$ (a suitable convex function $\nu$ is constructed as in the proof of Theorem $2(3)$ ). As it follows from Corollary $9, \widetilde{C}^{\prime}$ is a weak M-smoothing.

Remark 6 To check that $\widetilde{C}^{\prime}$ in the above proof is a weak M-smoothing one may count explicitly the corresponding $\nu$ and $l$ and verify simultaneously that they turn (7) into an equality. Note, first, that the number of those ovals in $\widetilde{C}_{\mathbb{R}}^{\prime}$ which come from the ovals of $F_{s}^{(i)}$ in $\left(\mathbb{R}^{*}\right)^{2}$ is

$$
\sum_{u, i} \#\left(\operatorname{Int}\left(Q_{u}^{(i)}\right) \cap \mathbb{Z}^{2}\right)-l^{\prime}=\#\left(\operatorname{Int}(\mathcal{D}) \cap \mathbb{Z}^{2}\right)-l^{\prime}=\frac{1}{2}(\mu-r+1)-l^{\prime}
$$

where $l^{\prime}$ counts how many polynomials as in Proposition 10 occur in the set $\left\{F_{u}^{(i)}\right\}_{s, i}$. Renumber the polynomials $F_{u}^{(i)}$ as $F^{(1)}, \ldots, F^{(N)}$ following the order of the polygons $Q_{u}^{(i)}$ from up downwards. Assume that $F^{\left(k_{i}\right)}, i=1, \ldots, l^{\prime}$, are as in Proposition 10. If two real branches $\mathcal{P}_{1}, \mathcal{P}_{2}$ of $\widetilde{C}_{\mathbb{R}}$ correspond to peripheral roots of $F^{(j)}, F^{(k)}$ such that $j<k_{i} \leq k$ for some $i=1, \ldots, l^{\prime}$, then neither of the endpoints of $\mathcal{P}_{1}$ on $B(C, z)_{\mathbb{R}}$ belongs to the same connected component of $\widetilde{C}_{\mathbb{R}}^{\prime}$ as an endpoint of $\mathcal{P}_{2}$. Indeed, if there were such a component, it would have passed in $Q_{k}^{(i)}$ from the edge $[(0, p),(q, p+1)]$ (or its symmetric image) to the edge $[(q, p+1),(q+s, 1)]$ or $[(0,0),(q+s, 1)]$ (or their symmetric images), but it is not the case as shown in Figure 18. Therefore, if one of $F^{(j)}$ with $j<k_{1}$ has a real peripheral root, then $l \geq 1+l^{\prime}$, which implies that (7) turns into an equality. If the polynomials $F^{(j)}$, $j=1, \ldots, k_{1}-1$, has only imaginary peripheral roots, then $l \geq l^{\prime}$. In this case the arc in $\mathrm{Ch}\left(F^{\left(k_{1}\right)}\right)$ shown in Figure 18 continued in the union of the charts of $F^{(j)}$, $j=1, \ldots, k_{1}-1$, gives one more oval in $\widetilde{C}_{\mathbb{R}}^{\prime}$ which together with previously counted ovals (10) and $l \geq l^{\prime}$ provides the equality in (7). 
1. Consider a bouquet of three real ordinary cusps with common vertex at 0 (each one topologically equivalent over $\mathbb{R}$ to $x^{2}-y^{3}=0$ ). In the case of distinct tangents, such singularities form two real topologically equisingular over $\mathbb{R}$ families: bouquets which are contained in a half-plane with the boundary line through 0 and those which are not. The singularities of the first class have M-smoothing, while, as shown in [9], the singularities of the second class (called in [9] Sirler singularities) have no M-smoothing. The proof given in [9] exploits various particular geometrical properties of Sirler singularities. So, is there any general geometric phenomenon behind the nonexistence of $M$-smoothing?

Look at intermediate positions of three cusps. The construction in the proof of Theorem 5 applied to a bouquet of 3 cusps with 2 different tangents, where the cusps with the same tangent are directed into opposite sides, shows that this singular point is deformation equivalent over $\mathbb{R}$ to a singularity which has $\mathrm{M}$-smoothing. What are topological and geometrical properties of the stratum of singularities which have $M$ smoothing? Say, in the versal deformation of a singular point?

As it follows from above examples, this stratum is not necessarily closed or open. However, in a $\mu$-constant families, i.e., inside families of real topologically equisingular over $\mathbb{R}$ singularities, the set of singularities which have M-smoothing is closed. Is it open? i.e., Is it true that the real singularities which are topologically equisingular over $\mathbb{R}$ either all have or all have no $M$-smoothing?

2. In view of Theorem 2 and the above question, it is natural to ask: Does any semi-quasi-homogeneous singular point admit an M-smoothing? Though the construction set up in the proof of Theorem 2 provides M-smoothings only for special values of the peripheral roots, the following example shows that the Viro theorem with a different subdivision of the domain under the Newton diagram can give Msmoothings for the whole range of the roots. The same example is the simplest singularity where the algorithm of [16] fails, i.e. the singularity which has M-smoothing but not BM-smoothing.

Example. For a singular point $\left(y^{2}+a x^{3}\right)\left(y^{2}+b x^{3}\right)=0$ with arbitrary $a<0<b$ there is a smoothing with $\frac{1}{2}(\mu-r+1)=7$ ovals.

Proof. First, construct a polynomial $F_{1}$ with $\Delta\left(F_{1}\right)=\operatorname{conv}\{(0,0),(0,2),(4,0)\}$ and the chart shown in Figure 19a. We get it by patch-working two polynomials: $F_{1}^{\prime}$, which has $\Delta\left(F_{1}^{\prime}\right)=\operatorname{conv}\{(0,2),(4,0),(3,0)\}$, truncation $\left(y+a x^{2}\right)\left(y+b x^{2}\right)$ on $[(0,2),(4,0)]$, and vertical tangent at the intersection point with the horizontal axis; and $F_{1}^{\prime \prime}$, which has $\Delta\left(F_{1}^{\prime \prime}\right)=\operatorname{conv}\{(0,0),(0,2),(3,0)\}$ and the chart as in Figure 19a. The shift $(x, y) \mapsto(x+\alpha, y)$ with a suitable $\alpha$ transforms $F_{1}$ in $F_{2}$, which has $\Delta\left(F_{2}\right)=$ $\operatorname{conv}\{(0,2),(1,0),(4,0)\}$, the same truncation on the edge $[(0,2),(4,0)]$ as $F_{1}$, and the chart as in Figure 19b. Consider the polynomial $F_{3}(x, y)=x^{-2} y^{4} F_{2}\left(x^{2} y^{-1}, x\right)$. It has $\Delta\left(F_{3}\right)=\operatorname{conv}\{(0,3),(0,4),(6,0)\}$ and its truncation on $[(0,4),(6,0)]$ is $\left(y^{2}+\right.$ $\left.a x^{3}\right)\left(y^{2}+b x^{3}\right)$. Its chart is shown in Figure $19 \mathrm{c}$. Patch-working $F_{3}$ with a polynomial, which has $\Delta\left(F_{3}\right)=\operatorname{conv}\{(0,0),(0,3),(6,0)\}$, the chart shown in Figure 19d and prescribed truncation with negative roots on $[(0,3),(6,0)]$ (such a polynomial is 
natural to ask is this version (or its stronger form, with $a=0$ ) of the Harnack-SmithThom inequality sharp? Recent results on the sharpness of the Harnack-Smith-Thom upper bound

$$
\operatorname{dim} H_{*}\left(X_{\mathbb{R}}, \mathbb{Z} / 2 \mathbb{Z}\right) \leq \operatorname{dim} H_{*}\left(X_{\mathbb{C}}, \mathbb{Z} / 2 \mathbb{Z}\right)
$$

for projective nonsingular hypersurfaces and complete intersections (Itenberg-Viro, unpublished) seem to show the sharpness of the above local version for homogeneous hypersurface and complete intersections singularities, up to equisingular deformation over $\mathbb{C}$.

\section{References}

[1] Arnold, V. I.: Some open problems in the theory of singularities In: Proc. Sobolev Seminar, Novosibirsk, (in Russian). English translation in Singularities./P. Orlik ed., Proceedings of symposia in Pure Math., Vol. 40, Part 1. Providence, AMS, 1983, p. 57-69.

[2] Arnold, V. I., Gusein-zade, S. M. and Varchenko, A. N.: Singularities of differentiable maps, vol.1, Birkhäuser, Boston etc., 1985.

[3] Baker, H. F.: Examples of the application of Newton polygon applied to the theory of singular points of algebraic functions, Math. Proc. Cambridge Phil. Soc. 15 (1893), 403-450.

[4] Chevalier, B.: Secteurs et déformations locales de courbes réelles. Math. Ann. 307 (1997), 1-28.

[5] Degtyarev, A. and Kharlamov, V.: Halves of a real Enriques surface. Comment. Math. Helvetici 71 (1996), 628-663.

[6] Harnack, A.: Über die Vielfaltigkeit der ebenen algebraischen Kurven. Math. Ann. 10 (1876), 189-199.

[7] Hironaka, H.: Arithmetic genera and effective genera of algebraic curves. $\mathrm{Mem}$. Coll. Sci. Univ. Kyoto. Sect. A30 (1956), 177-195.

[8] Kharlamov, V. and Viro, O.: Extensions of the Gudkov-Rohlin congruence. In: Rokhlin seminar (Lecture Notes in Math. 1345), Springer, Berlin etc, 1988, pp. $357-406$.

[9] Kharlamov, V., Orevkov, S., and Shustin, E.: Singularity which has no Msmoothing. To appear in Arnol'dfest (Proc. Intern. Conf. in honour of 60th birthday of V.I. Arnol'd, Toronto, June 1997), Fields Inst. Commun.

[10] Kharlamov, V., and Risler, J.-J.: Blowing-up construction of maximal smoothings of real plane curve singularities. In: Real Analytic and Algebraic Geometry. F. Broglia, M. Galbiati and A. Tognoli eds., (Proc. Intern. Conf. Trento (Italy), September 21-25, 1992), Walter de Gruyter, Berlin, New York, 1995, pp. 169188. 
[11] Khovanskii, A. G.: Newton polyhedra and the genus of complete intersections. Func. Anal. Appl. 12 (1978), no. 1, 35-43.

[12] Kouchnirenko, A. G.: Polyédres de Newton et nombres de Milnor. Invent. Math. 32 (1976), 1-31.

[13] Lê Dung Tràng and Ramanujam, C. P.: The invariance of Milnor's number implies the invariance of the topological type. Amer. J. Math. 98 (1976), no. $1,67-78$.

[14] Milnor, J.: Singular points of complex hypersurfaces. Annals of Math. Studies 61, Princeton Univ. Press, 1968.

[15] Pecker, D.: Sur le theoreme local de Harnack. C. R. Acad. Sci. Paris, Serie I, 326 (1998), no. 4, 573-576.

[16] Risler, J.-J.: Un analogue local du théoréme de Harnack. Invent. Math. 89 (1987), no. 1, 119-137.

[17] Shustin. E.: Smoothings of quasi-non-degenerate singularities. Preprint, Tel Aviv University, 1992.

[18] Swan, R.G.: A new method in fixed point theory. Bull. Amer. Math. Soc. bf 65 (1959), 128-130.

[19] Teissier, B.: The hunting of invariants in the geometry of discriminants. In: Real and Complex Singularities./ P.Holm, editor, Oslo: Sijthoff-Noordhoff Publ., Alphen aan den Rijn, 1977, p. 565-677.

[20] Teissier, B.: Résolution simultanée. In: Séminaire sur les singularités des surfaces (Lecture Notes in Math. 777), Springer, Berlin etc., 1980, pp. 72-146.

[21] Tougeron, J. C.: Ideaux des fonctions différentiables. Ann. Inst. Fourier (Grenoble) 18 (1968), no. 1, 177-240.

[22] Viro, O. Ya.: Gluing of algebraic hypersurfaces, smoothing of singularities and construction of curves. Proc. Leningrad Int. Topological Conf., Leningrad, Aug. 1983, Nauka, Leningrad, 1983, p. 149-197 (Russian).

[23] Viro, O. Ya.: Gluing of plane real algebraic curves and construction of curves of degrees 6 and 7. Lect. Notes Math. 1060, Springer, Berlin etc., 1984, p. 187-200. 